Chapter 1

\title{
The odderon and BKP states in the Quantum Chromodynamics
}

\author{
M.A.Braun \\ Dep. of High Energy physics, Saint-Petersburg State University, \\ 198504 S.Petersburg, Russia \\ G.P. Vacca \\ INFN Sezione di Bologna, Via Irnerio 46, 40126, Bologna, Italy
}




\begin{abstract}
We discuss the odderon in the QCD and analyze the effects of running coupling introduced in a particular way to preserve the reggeization of the gluon within the leading logarithmic description. This idea is also applied to a family of BKP states with arbitrarily number of gluons in the planar limit. The numerical analysis shows that contrary to the pomeron case where the leading states become discretized, the odderon states still remain a continuous family starting at intercept one. The following rapidity dependence of the amplitude is studied. For the pomeron-odderon system the relation between the descriptions in the reggeized gluon (BFKL) framework and the color dipole/CGC one is investigated.
\end{abstract}




\subsection{Introduction}

The odderon has a long history in the High-Energy physics. It started in 1973 and its first 30 years of existence are exposed in the comprehensive review 1]. So here we only mention some principal points and developments after 2003. The odderon was born as a result of an abstract idea that "strong interactions are as strong as they can be" in the paper by L. Lukaszuk and B. Nicolescu [2] and was given a name "maximal odderon".

The famous Froissart theorem establishes a limitation on the behavior of the scattering amplitudes governed by strong interactions as

$$
|A(s, t)|<C s \log ^{2} s, \quad C<\pi / m_{\pi}^{2} \simeq 62 m b n .
$$

In fact the amplitude can be split into $C=+1$ and $C=-1$ parts (or signatures $\xi= \pm)$ :

$$
A^{( \pm)}(s)=\frac{1}{2}[A(s) \pm A(-s)]
$$

and the long-standing Pomeranchuk theorem asserted

$$
\frac{A^{-}(s)}{A^{+}(s)}{ }_{s \rightarrow \infty} \rightarrow 0
$$

where the leading part of the $A^{(+)}$and $A^{(-)}$in the high energy limit is associated to the pomeron and to the odderon exchange, respectively. The results following from the Regge theory and based on the leading $\omega, \rho$-Regge trajectory in the $C=-1$ sector fully confirmed this rule. The $\omega, \rho$ intercept is $\alpha_{\rho, \omega}(0) \simeq 1 / 2$ so that the corresponding reggeon interchange leads to the amplitude $A^{(-)} \sim \sqrt{s}$, which is fully confirmed by the experiment.

The idea of the maximal odderon proclaimed that both amplitudes $A^{( \pm)}$take its maximal value at $s \rightarrow \infty$, with the only difference that $A^{(+)}(s)$ is positive imaginary and $A^{(-)}(s)$ real:

$$
A^{(+)}(s) \sim i s\left(\log ^{2} s-i \pi \log s\right), \quad A^{(-)} \sim s\left(\ln ^{2} s-i \pi \log s\right), \quad s>>m^{2} .
$$

Let us briefly recall some simple and evident properties of the maximal odderon amplitude 1.4 (at $t=0$ ). As mentioned it is mostly real. Its imaginary part is proportional to $s \log s$, which corresponds to the difference between the particleparticle and particle-antiparticle cross-sections growing like $\log s$ :

$$
\sigma_{a \bar{b}}(s)-\sigma_{a b} \sim \log s, \quad s>>m^{2} .
$$

Since the total cross-section for each of the two reactions grows like $\log ^{2} s$, the Pomeranchuk theorem is fulfilled in the sense

$$
\frac{\sigma_{a \bar{b}}(s)}{\sigma_{a b}(s)} \rightarrow 1, \quad s \rightarrow \infty .
$$

The most striking feature of the asymptotic odderon is a nonvanishing ratio of the real and imaginary parts of the amplitudes:

$$
\frac{\operatorname{Re} A(s)}{\operatorname{Im} A(s)} \sim \text { const, } s>>m^{2} .
$$


In the course of time and depending on the experimental situation these properties served as a dominant motive for and against the introduction of the odderon. We shall turn to the experimental evidence for the odderon in the end of this section.

In an attempt to realize this picture in the complex angular $j$-plane the authors were obliged to introduce rather exotic singularities. Addressing the interested author to the review 1 to see the details, we only mention that typically higher order poles at $j=1$ were introduced to generate the desired $\log (s)$ dependence. The authors had also to struggle against the singularities which might appear in the physical amplitude, since for $A^{(-)}$the point $j=1$ is physical.

With the advent of the Quantum Chromodynamics the odderon acquired a new life. In the lowest order there one finds both $C= \pm 1$ amplitudes on equal footing, the $C=+1$ one coming from the exchange of two gluons and the $C=-1$ from the exchange of three gluons. Both amplitudes are linear in $s$, so that their behavior at $s \rightarrow \infty$ is similar and corresponds to a pole in the $j$ plane at $j=1$. Ever since the theoretical efforts were directed to find corrections to this simple results in higher orders. Remarkable success has been achieved in the so-called leading$\log$ approximation, in which terms of the $\operatorname{order}\left(\alpha_{s} \ln (s)\right)^{n}$ are summed at high $s$ and small $\alpha_{s}$. A well-known BFKL equation was set up and solved to describe the evolution of the pomeron as early as $1975,3,4$ and its generalization to the next-to leading order in was derived in 1995 ,5, 6. The vertex for splitting of the pomeron in two was constructed in 1995 in two approaches, the dispersion one, following the derivation of the BFKL equation in [7] and in the approach based on the color dipole picture in 8. On the basis of this vertex equations for the scattering of a small object on the nucleus were proposed by I.I. Balitsky and Yu. Kovchegov (the BK equation) 9, 10, later generalized to CGC-JINWLK equation (see e.g. [11). Analogous developments were realized in the BFKL-Bartels approach 12:13]. Parallel to this the odderon was investigated both in the BFKL-Bartels and dipole approaches. The equation for the odderon in the leading-log approximation was set up as one of the so-called BKP equations proposed in 14 16. L.N. Lipatov analyzed the conformal properties of the odderon and related its equation to that for the solvable chain of conformal spins [17]. This allowed to R.A. Janik and J. Wosiek to obtain an explicit equation for the odderon wave function (the JW odderon) and find its maximal intercept 18

$$
\alpha_{O}=1-0.24717 \frac{\alpha_{s} N_{c}}{\pi},
$$

where $\alpha_{s}=g_{s}^{2} /(4 \pi)$. Somewhat later a new solution of the odderon equation was obtained by J. Bartels, L.N. Lipatov and G.P. Vacca (the BLV odderon) with $\alpha_{O}=1$ in which two of the three reggeons forming the odderon are fused into one [19]. This simple odderon was later also found in the dipole approach in [20]. On the basis of the latter the evolution equation was set up for the combined pomeron-odderon system in [21 and attempts at solving it were made in 21 , 22], although in a very simplified (one-dimensional) approach. As to the more 
complicated JW odderon, the necessary pomeron-two odderon vertex was found in 23], although its application has not been attempted so far, given that these odderon states where shown to decouple from some perturbative impact factors, while the BLV Odderon states are maximally coupled to them 24]. Later, using the effective action for reggeized gluon constructed by L. N. Lipatov, the BKP kernel for three reggeized gluon systems in the NLL approximation was constructed 25]. This can be used to analyze the odderon both in QCD, but in the large $N_{c}$ limit, or in general supersymmetric extensions, in particular $N=4 \mathrm{SYM}$ for any $N_{c}$. Then, starting from generalized bootstrap equations involving inelastic production amplitudes, which were conjectured 26] but have still to be proved, a construction for a family of odderon solution at NLL was given. They are extensions of the BLV solution to the NLL approximation and have also intercept starting strictly at one 26.

We stress that in all developments of the odderon theory seminal contributions were made by L.N. Lipatov, beginning from the study of the BFKL pomeron and including the understanding of the conformal properties of the pomeron and odderon, the equivalence of the odderon equation to the solvable chain of conformal spins and construction of the BLV odderon.

On the experimental side the history of the odderon is just as long but marked by much less clarity, novelty and success. Manifestations of the odderon can be present either in the processes realized by the purely odderon exchange or in the interference of the pomeron and odderon exchanges. The typical example of the first case is the production of a PS meson $(\mathrm{C}=+1)$ in the interactions starting with the photon $(\mathrm{C}=-1)$ either real or virtual. Discovery of this process would be a straightforward detection of the odderon. There were various theoretical estimates of the rate of this transition e.g. for the process $\gamma \rightarrow \eta_{c}$ [24,27] (see also the review 1] for earlier works). Unfortunately they all gave estimations for the cross-sections far below the present experimental possibilities. More informative have been attempts to see the interference of the pomeron and odderon exchanges primarily in the comparison of $p p$ and $p \bar{p}$ elastic scattering, In fact this comparison was the basic motivation for the introduction of the odderon in the first papers in which this notion was introduced. Already at that time (in the seventies) a significant difference between these two cross-sections was discovered. In particular the dip present in the $p p$ cross-section as a function of $t$ was not seen in the $p \bar{p}$ cross-section. At comparatively low energies this difference could be satisfactorily explained by the exchange of the well-known $C=-1$ reggeons $\omega-\rho$ with an intercept much below unity. However the difference did not disappear at higher energies. The situation at present date can be seen from Fig. 1.2 in which the latest data on the elastic $p p$ scattering data from the TOTEM collaboration at $2.76 \mathrm{TeV}[28$ are compared to the $p \bar{p}$ cross-sections from $\mathrm{D} 0$ at $1.96 \mathrm{Tev}$ 29. Remarkably the picture is nearly identical to the similar one for the experiments at $53 \mathrm{GeV}$ presented in the review [1] in 2003. So the difference between $p p$ and $p \bar{p}$, if indeed it exists, does not depend on energy once it 
is high enough. This latest experiments renewed extensive discussion of the odderon contribution at high energies, with not, however, conclusive arguments, especially in view of considerable experimental uncertainties 30,32 , not to consider still open theoretical issues.

In the present paper we try to cover some theoretical questions left untouched in the previous studies, which may throw additional light on the odderon as the QCD object. In the first place we consider interacting pomeron-odderon systems in QCD in the generalized leading logarithmic approximation to discuss the link between the reggeized gluon BFKL approach and the findings in the dipole/CGC picture. In particular we try to construct the equations for the combined evolution of the pomeron, and BLV odderon in the dense (nuclear) target in the BFKL-Bartels approach to compare with similar equations in the dipole/CGC picture 21. We shall be able to derive only the first of these equations: evolution of the pomeron in the presence of the odderon field. The second equation for the odderon itself requires knowledge of the vertex for the transition of three reggeons to five reggeons, the task not realized so far and evidently beyond the scope of this study. We hope to return to this problem in future. We also discuss what one may think could be an effective useful description in the non perturbative regime and in the large transverse distance limit, the so called Reggeon Field Theory developed by V.Gribov for which we review some details of the universal critical behavior. Then we study the influence of the running coupling on the odderon and higher BKP states, which was not given much attention in the past. We also present in this framework the numerical study for both the pomeron and the odderon spectrum.

\subsection{Interacting pomeron-odderon systems}

We start discussing the perturbative QCD behavior of pomeron-odderon system, interactions which go in the direction of "unitarize" the theory in the sense of removing the violation of the Froissart bound. Full evolution of effective interacting pomeron-odderon systems is too hard to be investigated in small $\mathrm{x} Q \mathrm{QCD}$, since it should involve also loops. Nevertheless an effective tree level description has been used to study some features of the large rapidity evolution and propagation in a dense nuclear medium. A simplified model, the so called Reggeon Field Theory is subsequently presented. Computation with loops are at reach there and we give some recent perturbative results for the critical properties.

\subsubsection{Fan diagrams for the pomeron-odderon system}

The distribution of gluons (and quarks) in hadronic scattering processes depends on the rapidity under consideration and can be associated to phases characterized by high densities of strongly interacting quanta. Collisions with the center of mass energy dominating over other momentum scales require summation of contributions proportional to powers of $\log s$. This task was realized by L.N. Lipatov and 


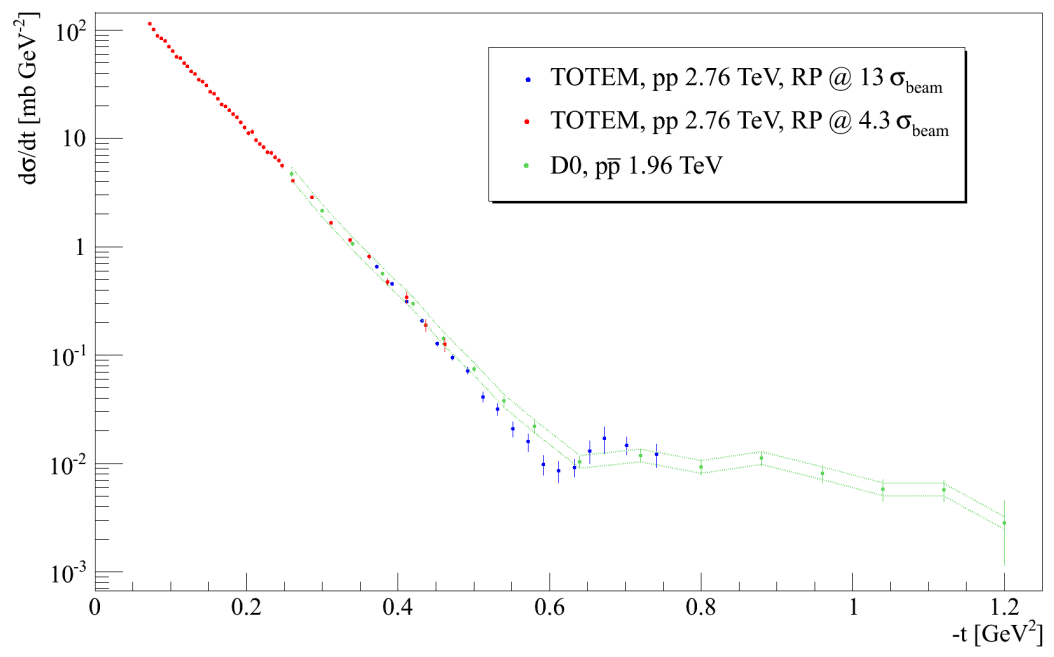

Fig. 1.1 The differential cross sections $\mathrm{d} \sigma / \mathrm{d} t$ at $\sqrt{s}=2.76 \mathrm{TeV}$ measured by the TOTEM experiment and the elastic p $\overline{\mathrm{p}}$ measurement of the D0 experiment at $1.96 \mathrm{TeV}[29$. The green dashed line indicates the normalization uncertainty of the D0 measurement.

collaborators in the so called BFKL approach. The leading log (LL) and next-toleading-log (NLL) approximations resum $\left(\alpha_{s} \log s\right)^{2}$ and $\alpha_{s}\left(\alpha_{s} \log s\right)^{2}$ contributions, respectively. In the lowest order of perturbation theory it is sufficient to take into account only two reggeized gluons exchanged in the colorless $t$-channel. Then it was discovered that the cross sections grow as a power of energy in violation of the unitarity restrictions. This clearly showed the necessity to include more complicated structures into the $t$-channel, larger numbers of reggeized gluons and transitions changing these numbers. Of course the final answer to the problem requires solving fully the Quantum Chromodynamics in the relevant kinematical conditions (Regge kinematics), which does not seem realistic. So at present one has to rely on certain approximations starting from the simplest BFKL approach. Their improvememnt can be realized by different simplified models based on diagrams constructed from reggeized gluons in planar approximation or from pomeron diagrams without loops.

Considerable progress has been achieved in the study of the scattering of compact objects , quark-antiquark loops or "onia". One starts from the scattering amplitudes for the collision of two such objects. In the BFKL approach it can be factorized into impact factors (local in rapidity) and a BFKL pomeron Green function where the rapidity dependence is encoded, both objects depending on the transverse momenta 
$\boldsymbol{q}$ and $\boldsymbol{k}$

$$
\mathcal{A}(Y, \boldsymbol{q})=i \int d^{2} \boldsymbol{k} \Phi_{1}(\boldsymbol{q}, \boldsymbol{k}) G_{Y}(\boldsymbol{q}, \boldsymbol{k}) \Phi_{1}(\boldsymbol{q}, \boldsymbol{k}),
$$

The Green function $G_{Y}$ is associated to the evolution kernel later described in Section 1.3.2 restricting to a fixed coupling constant. Including more $t$ channel gluons in the analysis and taking into account the change of their numbers has been a long lasting program started by J. Bartels. Essentially the analysis was pushed up to six $t$-channel reggeized gluons. It was based on the study of multicut amplitudes $D_{n}$ for the transition of the quark-antiquark loop into $n$ reggeized gluons (actually their multiple energy discontinuities). They can be related to the total cross sections by the AGK rules [33, 34]. A set of coupled equations has been constructed for $D_{n}$ with the following ingredients: impact factors $D_{n}^{0)}$ for $n$ reggeized gluons directly attached to the quark-antiquark loop, the reggeized gluon trajectories, the $K_{n \rightarrow m}$ elementary transition vertices from $n$ to $m$ reggeized gluons and the so-called bootstrap relations.

As the outcome, the amplitude $D_{n}$ can be presented as a sum two contributions, the so-called reggeized one $D_{N}^{R}$ for which evolution in rapidity is governed by a single pomeron Green function for two reggeized gluons $G_{2}$ (BFKL Green function), and the other, irreducable one, $D_{n}^{I}$ which evolves in rapidity via Green functions $G_{n}$ for $n>2$ reggeized gluons. In particular for the 4-gluon amplitude one finds

$$
D_{4}=D_{4}^{R}+D_{4}^{I} \quad, D_{4}^{I}=\int G_{4}\left(Y-Y^{\prime}\right) V_{2 \rightarrow 4} D_{2}\left(Y^{\prime}\right)=G_{4} \otimes V_{2 \rightarrow 4} D_{2},
$$

where $D_{2}\left(y^{\prime}\right)=G_{2}\left(y^{\prime}\right) D_{2}(0)$ and the $V_{2 \rightarrow 4}$ vertex describes the transition from 2 to 4 reggeized gluons and $G_{4}$ is the Green function for the general evolution of 4 reggeized gluons. Similarly for the 6-gluon amplitude one finds 23

$$
D_{6}=D_{6}^{R}+D_{6}^{I}+D_{6}^{E}
$$

where

$$
D_{6}^{I}=\int G_{6}\left(Y-Y^{\prime}\right) V_{2 \rightarrow 6} D_{2}\left(Y^{\prime}\right)=G_{6} \otimes V_{2 \rightarrow 6} D_{2} .
$$

It is remarkable that in the vertex $V_{2 \rightarrow 6}$ a term appears with a colour structure containing a product of two coefficients $d_{a b c}$. It describes transition of a pomeron into two odderons. The last term

$$
D_{6}^{E}=\sum G_{4} \otimes V_{2 \rightarrow 4} G_{4} \otimes V_{2 \rightarrow 4} D_{2}+D^{e x t r a}
$$

contains contributions related to a double $2 \rightarrow 4$ splitting including the BKP evolution (fan structure) and finally another term with a peculiar tensor color structure which was not completely analyzed 23 .

In an alternative, dipole or CGC approach, started by A.H. Mueller with collaborators, the scattering of an "onium" on a dense nuclear target was described (in the large $N_{C}$ limit) by the Balitski-Kovchegov evolution equation 9, 10. From 
the viewpoint of the BFKL-Bartels approach this equation is equivalent to summation of fan diagrams made of BFKL green functions and triple pomeron vertices $\left.V_{2 \rightarrow 4} 35\right]$.

From the start in the BFKL theory apart from the pomeron also the odderon was introduced as a compound state of three reggeized gluons with a color factor $d_{a b c}$. In its general form its wave function depends on the three gluon momenta (or coordinates). The equation for this wave function was studied by L.N. Lipatov [17 and, as mentioned in the Introduction, later solved by Janik and Woiscek (JWodderon) who showed that its ground state has an intercept smaller than unity. So the JW-odderon generates cross-sections vanishing at high energies.

However later a different odderon state was found, which essentially depends on only two coordinates with a fusing pair of the three gluons, the BLV-odderon [19. Its wave function is essentially the antisymmetric pomeron wave function whose ground state has the intercept exactly equal to one. Contribution from the BLV-odderon remains constant at high energies and so dominate over the JWodderon. Moreover some leading order impact factors are known to decouple from JW-odderon while couple maximally with the BLV-odderon [24. Explicitly the BLV-odderon eigenstate $\Psi$ is written in terms of the odd pomeron eigenstate $\psi$ as

$$
\Psi=\mathcal{N} \hat{S} \psi
$$

where $\hat{S}$ is an operator acting on 2 -gluon states and with values on the 3 -gluon states, which performs an antisymmetrization in the 2 incoming gluons, splits the first of them in two and sums over the cyclic permutations of the outgoing gluons:

$$
\hat{S}\left(1,2,3 \mid 1^{\prime}, 2^{\prime}\right) \phi\left(1^{\prime}, 2^{\prime}\right)=\frac{1}{2} \sum_{(123)}[\phi(12,3)-\phi(3,12)] .
$$

and $\mathcal{N}$ is a normalization factor. One finds

$$
\langle\Psi \mid \Psi\rangle=c_{o}\left\langle\psi\left|\mathcal{N}^{2} H_{12}\right| \psi\right\rangle,
$$

where $c_{o}=3 g_{s}^{2}\left(N_{c}^{2}-4\right) / N_{c}^{2}$. If one wants to have the same normalized scalar product for $\Psi$ as for $\psi$ then one has to choose $\mathcal{N}=\left(c_{o} H_{12}\right)^{-1 / 2}$.

Somewhat later this odderon was also found in the dipole approach [20] also associated with the antisymmetric pomeron state. Subsequently this dipole odderon was included into the fan diagrams giving rise to the evolution equations for the combined pomeron plus odderon evolution. Denoting the pomeron and odderon density fields as $N$ and $O$ respectively, the equations are 21.

$$
\begin{aligned}
& \frac{d}{d Y} N_{\boldsymbol{x}, \boldsymbol{y}}=\bar{\alpha}_{s} \int \frac{d^{2} z}{2 \pi} \frac{|x-y|^{2}}{|x-z|^{2}|y-z|^{2}} \times \\
& \left(N_{\boldsymbol{x}, \boldsymbol{z}}+N_{\boldsymbol{y}, \boldsymbol{z}}-N_{\boldsymbol{x}, \boldsymbol{y}}-N_{\boldsymbol{x}, \boldsymbol{z}} N_{\boldsymbol{y}, \boldsymbol{z}}+O_{\boldsymbol{x}, \boldsymbol{z}} O_{\boldsymbol{y}, \boldsymbol{z}}\right) . \\
& \frac{d}{d Y} O_{\boldsymbol{x}, \boldsymbol{y}}=\bar{\alpha}_{s} \int \frac{d^{2} z}{2 \pi} \frac{|x-y|^{2}}{|x-z|^{2}|y-z|^{2}} \times \\
& \left(O_{\boldsymbol{x}, \boldsymbol{z}}+O_{\boldsymbol{y}, \boldsymbol{z}}-O_{\boldsymbol{x}, \boldsymbol{y}}-O_{\boldsymbol{x}, \boldsymbol{z}} N_{\boldsymbol{y}, \boldsymbol{z}}-N_{\boldsymbol{x}, \boldsymbol{z}} O_{\boldsymbol{y}, \boldsymbol{z}}\right) \text {. }
\end{aligned}
$$


Here we show that at least the first of these equations can be derived in the BFKL-Bartels framework on the basis of the known transitional vertex $V_{2 \rightarrow 6}$ constructed in 23 .

First we recall how the pomeron part of Eq. (1.16) was derived in [35]. The linear part on the right hand side of both equations (1.16) and (1.17) is just the BFKL evolution of the pomeron and odderon fields. To see the link between the reggeized gluon BFKL description and the dipole picture consider the evolution of the BFKL pomeron wave function in the form of the quasi-Schroedinger equation

$$
\frac{d}{d Y} \psi\left(\rho_{1}, \rho_{2}\right)=-H_{12} \psi\left(\rho_{1}, \rho_{2}\right)
$$

with the kernel $H_{12}=\frac{\bar{\alpha}_{s}}{2} h_{12}$, where $\bar{\alpha}_{s}=\alpha_{s} N_{c} / \pi$ and

$$
h_{12}=\ln \left|p_{1}\right|^{2}+\ln \left|p_{2}\right|^{2}+\frac{1}{p_{1} p_{2}^{*}} \ln \left|\rho_{12}\right|^{2} p_{1} p_{2}^{*}+\frac{1}{p_{1}^{*} p_{2}} \ln \left|\rho_{12}\right|^{2} p_{1}^{*} p_{2}-4 \Psi(1) .
$$

Here $\Psi(x)=d \ln \Gamma(x) / d x$, gluon holomorphic momenta are used and the first two log terms belong to the virtual corrections. Passing to the coordinate representation and taking into account that the coordinate wave function of the pomeron vanishes when the two reggeized gluon are located at the same point (termed "the Möbius representation in 35,36 ") the action of this kernel on a state can be rewritten as

$$
H_{12} \psi\left(\boldsymbol{\rho}_{1}, \boldsymbol{\rho}_{2}\right)=\bar{\alpha}_{s} \int \frac{d^{2} \rho_{3}}{2 \pi} \frac{\left|\rho_{12}\right|^{2}}{\left|\rho_{13}\right|^{2}\left|\rho_{23}\right|^{2}}\left(\psi\left(\boldsymbol{\rho}_{1}, \boldsymbol{\rho}_{2}\right)-\psi\left(\boldsymbol{\rho}_{1}, \boldsymbol{\rho}_{3}\right)-\psi\left(\boldsymbol{\rho}_{2}, \boldsymbol{\rho}_{3}\right)\right),
$$

This has the same form as the linear part of Eqs. 1.16 and (1.17).

Now consider the first non-linear term on the right-hand side of Eq. (1.16). The irreducible term $D_{4}^{I}$ of Eq. 1.9 contains the transition from 2 to 4 reggeized gluon states. The latter evolve in rapidity with the 4-gluon Green function, which in the planar (large $N_{c}$ ) limit factorizes into the product of two independent BFKL Green functions: $G_{4}=G_{2} \times G_{2}$. Then the second term of Eq. (1.9) acquires the structure of the triple pomeron vertex. In the planar limit $V_{2 \rightarrow 4} D_{2}$ becomes

$$
\begin{aligned}
& V(1234) D_{2}=\frac{1}{2} g^{2}[G(1,2+3,4)+G(2,1+3,4)+G(1,2+4,3)+G(2,1+4,3) \\
& -G(1+2,3,4)-G(1+2,4,3)-G(1,2,3+4)-G(2,1,3+4)+G(1+2,0,3+4)]
\end{aligned}
$$

where number $i$ stands from the momentum $q_{i}$. The general function $G(1,2,3)$ was defined in 37] and consists of two pieces

$$
G\left(\boldsymbol{k}_{1}, \boldsymbol{k}_{2}, \boldsymbol{k}_{3}\right)=G_{1}\left(\boldsymbol{k}_{1}, \boldsymbol{k}_{2}, \boldsymbol{k}_{3}\right)+G_{2}\left(\boldsymbol{k}_{1}, \boldsymbol{k}_{2}, \boldsymbol{k}_{3}\right) .
$$

The first term is constructed from real diagrams

$$
G_{1}\left(\boldsymbol{k}_{1}, \boldsymbol{k}_{2}, \boldsymbol{k}_{3}\right)=g^{2} N_{c} \int \frac{d^{2} \boldsymbol{q}_{1} d^{2} \boldsymbol{q}_{2}}{(2 \pi)^{3}} \delta^{2}\left(\boldsymbol{q}_{1}+\boldsymbol{q}_{2}-\boldsymbol{q}\right) W\left(\boldsymbol{k}_{1}, \boldsymbol{k}_{2}, \boldsymbol{k}_{3} \mid \boldsymbol{q}_{1}, \boldsymbol{q}_{2}\right) D_{2}\left(\boldsymbol{q}_{1}, \boldsymbol{q}_{2}\right),
$$


where $\boldsymbol{q}=\boldsymbol{k}_{1}+\boldsymbol{k}_{2}+\boldsymbol{k}_{3}$ and

$$
W\left(\boldsymbol{k}_{1}, \boldsymbol{k}_{2}, \boldsymbol{k}_{3} \mid \boldsymbol{q}_{1}, \boldsymbol{q}_{2}\right)=\left(\frac{\left(\boldsymbol{k}_{2}+\boldsymbol{k}_{3}\right)^{2}}{\left(\boldsymbol{q}_{1}-\boldsymbol{k}_{1}\right)^{2} \boldsymbol{q}_{2}^{2}}+\frac{\left(\boldsymbol{k}_{1}+\boldsymbol{k}_{2}\right)^{2}}{\boldsymbol{q}_{1}^{2}\left(\boldsymbol{q}_{2}-\boldsymbol{k}_{3}\right)^{2}}-\frac{\boldsymbol{k}_{2}^{2}}{\left(\boldsymbol{q}_{1}-\boldsymbol{k}_{1}\right)^{2}\left(\boldsymbol{q}_{2}-\boldsymbol{k}_{3}\right)^{2}}-\frac{\boldsymbol{q}^{2}}{\boldsymbol{q}_{1}^{2} \boldsymbol{q}_{2}^{2}}\right)
$$

The second term is related to the virtual correction present in the reggeized gluon trajectory:

$$
\begin{aligned}
G_{2}\left(\boldsymbol{k}_{1}, \boldsymbol{k}_{2}, \boldsymbol{k}_{3}\right)= & -\left[\omega\left(\boldsymbol{k}_{2}\right)-\omega\left(\boldsymbol{k}_{2}+\boldsymbol{k}_{3}\right)\right] D_{2}\left(\boldsymbol{k}_{1}, \boldsymbol{k}_{2}+\boldsymbol{k}_{3}\right) \\
& -\left[\omega\left(\boldsymbol{k}_{2}\right)-\omega\left(\boldsymbol{k}_{1}+\boldsymbol{k}_{2}\right)\right] D_{2}\left(\boldsymbol{k}_{1}+\boldsymbol{k}_{2}, \boldsymbol{k}_{3}\right) .
\end{aligned}
$$

Fourier transforming the vertex (1.21), $D_{2}$ and the two pomeron subsystems $G_{2}(12) \times G_{2}(34)$ (in fact passing to the Möbius representation for the states) one finds

$$
\begin{gathered}
T_{4} \equiv\left\langle\psi(12)\left|\left(V(1234) D_{2}\right)\right| \psi(34)\right\rangle=2 g_{s}^{2}\langle\psi(12)|(G(1,2+3,4)|\psi(34)\rangle \\
=\psi\left(\rho_{13}\right) \psi\left(\rho_{23}\right)\left[-c \frac{\rho_{12}^{2}}{\rho_{13}^{2} \rho_{23}^{2}}\right] D_{2}\left(\rho_{12}\right),
\end{gathered}
$$

where $c=g_{s}^{4} N_{c} /\left(4 \pi^{3}\right)$ and $\rho_{12}=\rho_{1}-\rho_{2}$. This has exactly the same form of the interaction term present in Eq. 1.16, with a suitable rescaling (by $\frac{\bar{\alpha}_{s}}{2 \pi c}$ ) of the two dipole (pomeron) fields $N\left(\rho_{1}, \rho_{3}\right) N\left(\rho_{2}, \rho_{3}\right)$.

Now we perform a similar derivation for the last term on the right-hand side, made of two odderon fields. It comes from the $V_{2 \rightarrow 6}$ vertex present in the $D_{6}^{I}$ of Eq. (1.11) discussed before. Consider the six gluons (1-6) grouped into two odderon fields as (123) and (456). In the planar limit the odderon term of the vertex can be written in a compact notation 27 as

$$
W_{6}^{\text {odd }}(1,2,3 \mid 4,5,6)=-\frac{1}{8} g_{s}^{4}\left(\hat{S}_{1}-\hat{P}_{1}\right) f_{12}\left(\hat{S}_{2}^{\dagger}-\hat{P}_{2}^{\dagger}\right)
$$

Here the indices 1 and 2 refer to the triplets of gluons (123) and (456), the function $f_{12}$ depends on four gluon momenta

$$
f(1,2 \mid 3,4)=G(1,2+3,4)-G(2,1+3,4)-G(1,2+4,3)+G(2,1+4,3)
$$

operator $\hat{S}$ was defined in 1.14 and finally $\hat{P}$ is an operator which acting on a function of two gluon momenta antisimmetrizes it in them and splits the first momentum in three outgoing momenta, while putting to zero the second one:

$$
\left.\left.\hat{P}\left(1,2,3 \mid 1^{\prime}, 2^{\prime}\right)\right) \phi\left(1^{\prime}, 2^{\prime}\right)=\frac{1}{2}[\phi(123,0))-\phi(0,123)\right] .
$$

(The presence of the subtraction term $(\hat{P})$ in the definition of $W_{6}^{\text {odd }}$ is due to the fact that the function $f_{12}$ is not zero in the two 2-gluons subsectors when one the momenta is zero). Calling $\varphi_{-}$a generic two gluon antisymmetric function one has 19

$$
\left\langle\Psi \mid(\hat{S}-\hat{P}) \varphi_{-}\right\rangle=c_{o}\left\langle\psi\left|\mathcal{N} H_{12}\right| \varphi_{-}\right\rangle .
$$


Note that in the odderon channel the eigenvalues of $H_{12}$ are non negative. The BLV states start with the intercept exactly equal to unity.

One then finds

$$
\left\langle\Psi_{n, \nu}(1,2,3)\right|(\hat{S}-\hat{P})=c_{o}^{1 / 2}\left\langle\psi_{n, \nu}(1,2)\right| H_{12}^{1 / 2},
$$

where $n, \nu$ are the conformal quantum numbers of the odderon state $\Psi$ and the related pomeron state $\psi$. Using 1.30 the contraction of the vertex $W_{6}^{\text {odd }}$ with two odderon states $\Psi_{1}(1,2,3)$ and $\Psi_{2}(4,5,6)$ normalized as described above becomes

$$
\begin{gathered}
T_{6} \equiv-\frac{g_{s}^{2}}{8}\left\langle\Psi_{1}\left|\left(\hat{S}_{1}-\hat{P}_{1}\right) f_{12}\left(\hat{S}_{2}^{\dagger}-\hat{P}_{2}^{\dagger}\right)\right| \Psi_{2}\right\rangle \\
=-\frac{g_{s}^{2}}{8} c_{o}\left\langle\psi_{1 \nu_{1}, n_{1}}\left|H_{12}^{1 / 2} f_{12} H_{12}^{1 / 2}\right| \psi_{2 \nu_{2}, n_{2}}\right\rangle \\
=-\frac{g_{s}^{2}}{2} c_{o} \psi_{\nu_{1}, n_{1}}(1,2) H_{12}^{1 / 2} G(1,2+3,4) H_{34}^{1 / 2} \psi_{\nu_{2}, n_{2}}(3,4),
\end{gathered}
$$

where for the last equality the antisymmetry of the 2 gluon state $\psi$ associated to the odderon state is used. Then performing a Fourier transform one finally finds, similarly to Eq. 1.25,

$$
T_{6}=\psi\left(\rho_{13}\right) H_{13}^{1 / 2} \psi\left(\rho_{23}\right) H_{23}^{1 / 2}\left[c^{\prime} \frac{\rho_{12}^{2}}{\rho_{13}^{2} \rho_{23}^{2}}\right] D_{2}\left(\rho_{12}\right),
$$

where $c^{\prime}=\frac{g_{s}^{4} N_{c}}{16 \pi^{3}} c_{o}$ and one can identify in the square brackets the pomeron into two odderon vertex in the coordinate representation, which in Eq. 1.16 is multiplied

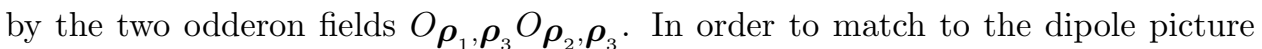
representation in the large $N_{c}$ limit, as before one needs to renormalize the BLV odderon field, rescaling it by the extra operatorial factor $H_{i j}^{1 / 2}$ as well by the constant factor $\frac{\bar{\alpha}_{s}}{2 \pi \sqrt{c c^{\prime}}}$.

It is remarkable and important that the pomeron-two-odderons vertex turns out to be of the opposite sign compared to the triple pomeron vertex. In this framework this fact comes automatically from the expressions of the two vertices $V_{2 \rightarrow 4}$ and $V_{2 \rightarrow 6}$. This property is related to the different signatures of the pomeron and odderon fields and in particular to the fact that in a pomeron amplitude the two pomeron cut is negative while the two odderon cut is positive. In the next subsection we show how these properties are realized in an effective local description called Reggeon Field Theory.

In the other Eq. (1.17) the interaction term $O N$ and $N O$ could be computed in the reggeized gluon approach starting from the transition vertex $V_{3 \rightarrow 5}$, which unfortunately has not been computed yet in the reggeized gluon approach. 


\subsubsection{A simplified picture: the Reggeon Field Theory}

The equations above are used to study pomeron-odderon fields in the regime where saturation effects set in taming their growth with rapidity. This behavior is induced by the fan structure which is resummed by the differential equations. Nevertheless, as always stressed by L.N. Lipatov, the real picture is more complicated since one should include also loops involving pomeron and odderon fields (and more in general reggeized gluons). Moreover at high energy (large rapidities) but long transverse distances non perturbative QCD physics, where hadrons are involved, cannot be ignored. This is a problem whose solution is well out of reach at present state.

Before the QCD era strong interactions were studied in the context of S-matrix and the scattering in Regge limit was described by simpler systems derived from the analytic structure of the partial wave amplitudes, in particular focusing on the so called reggeons associated to their poles. As the next natural step, reggeon interactions were considered by V.N. Gribov and encoded in a $2+1$ dimensional Reggeon Field Theory, with one time (rapidity) and two transverse space dimensions. This is a framework were pomeron and odderon interactions can be studied, in particular using renormalization group ( $R G$ ) techniques. Recently an investigation using a functional RG approach was carried on 38, 39, including the case of a system of one pomeron and one odderon local fields, denoted as $\psi, \psi^{\dagger}$ and $\chi, \chi^{\dagger}$ respectively. The RFT action in its simpler version, with just ultra local cubic interactions, has the form:

$$
\begin{aligned}
S\left[\psi^{\dagger}, \psi, \chi^{\dagger}, \chi\right]= & \int \mathrm{d}^{D} x \mathrm{~d} \tau\left(Z_{P}\left(\frac{1}{2} \psi^{\dagger} \stackrel{\leftrightarrow}{\partial}_{\tau} \psi-\alpha_{P}^{\prime} \psi^{\dagger} \nabla^{2} \psi\right)+Z_{O}\left(\frac{1}{2} \chi^{\dagger} \stackrel{\leftrightarrow}{\partial}_{\tau} \chi-\alpha_{O}^{\prime} \chi^{\dagger} \nabla^{2} \chi\right)\right. \\
& \left.+V\left[\psi, \psi^{\dagger}, \chi, \chi^{\dagger}\right]\right)
\end{aligned}
$$

with the potential $V=V_{3}$ given by

$$
V_{3}=-\mu_{P} \psi^{\dagger} \psi+i \lambda \psi^{\dagger}\left(\psi+\psi^{\dagger}\right) \psi-\mu_{O} \chi^{\dagger} \chi+i \lambda_{2} \chi^{\dagger}\left(\psi+\psi^{\dagger}\right) \chi+\lambda_{3}\left(\psi^{\dagger} \chi^{2}+\chi^{\dagger^{2}} \psi\right) \text {. }
$$

Here $D$ is the number of spatial dimension $(D=2$ is the physical case), which can be conveniently considered as a continuous parameter. The potential (allowed interactions) is constrained by signature conservation (even for the Pomeron and odd for the Odderon) and by the overall sign of the multi-Reggeon discontinuity amplitudes $-i \prod_{j}\left(i \xi_{j}\right)$, where $\xi_{j}$ are the signature factors $\xi=\left(\tau-e^{-i \pi \omega}\right) / \sin \pi \omega$ with $\omega=\alpha(0)-1$ : note that for the Pomeron $\xi_{P}$ is almost imaginary while for the Odderon $\xi_{O}$ is almost real. This implies that $t$-channel states with odd and even number of Odderons never mix. Another constraint on the potential is that transitions $P \rightarrow P P$ are imaginary (two Pomeron cut is negative), $P \rightarrow O O$ are real (two Odderon cut is positive) and $O \rightarrow O P$ is imaginary (Odderon-Pomeron cut is negative). These considerations, implemented in Eq. 1.34, are easily generalized to higher order (subleading) interactions [39]. As a consequence we can write the potential in terms of different contributions with operators which relate states differing by an integer number of Odderon pairs. In perturbative QCD the $P \rightarrow O O$ vertex has been computed in the generalized leading-log approximation. 
We shall see that the Reggeon interaction nevertheless are dominated by a fixed point in which operators changing the number of Odderon pairs are not present, these being present only in deformations of the critical theory.

Since $D=4$ is the scaling dimension (critical dimension) of reggeon field theory, critical behaviour can be studied in perturbation theory with the $\epsilon$ expansion in $D=4-\epsilon$ dimensions. Moreover functional renormalization group techniques based on the use of the effective average action can be used in $D=2$ in a truly non perturbative context. We present here the results of a one-loop renormalization group analysis in $D=4-\epsilon$ around the critical dimension 4 of the transverse space. We do not report the beta functions here but just the results for the fixed points and the linear behavior around them. In 39 also the case $D=2$ was studied using non perturbative methods based on functional renormalization group techniques, which shows that the perturbative results are qualitatively correct but quantitatively different. Using the cubic truncation one finds, besides a fixed point solution related to the pure Pomeron theory, a second non trivial fixed point also in the Odderon sector, such that $\lambda^{2}, \lambda_{2}^{2}, \lambda_{3}^{2}, \mu_{P}, \mu_{O}=O(\epsilon)$ :

$$
\begin{aligned}
& \mu_{P}=\frac{\epsilon}{12}, \quad \mu_{O}=\frac{95+17 \sqrt{33}}{2304} \epsilon, \quad \lambda^{2}=\frac{8 \pi^{2}}{3} \epsilon, \quad \eta_{P}=-\frac{\epsilon}{6}, \quad \eta_{O}=-\frac{7+\sqrt{33}}{72} \epsilon \\
& \lambda_{2}^{2}=\frac{23 \sqrt{6}+11 \sqrt{22}}{48} \epsilon, \quad \lambda_{3}=0, \quad \zeta_{P}=\zeta_{O}=\frac{\epsilon}{12}, \quad r=\frac{3}{16}(\sqrt{33}-1) .
\end{aligned}
$$

Here $\eta_{P, O}=-\mu \frac{d}{d \mu} \log Z_{P, O}$ are the corresponding field anomalous dimensions, $\zeta_{P, O}=-\mu \frac{d}{d \mu} \log \alpha_{P, O}^{\prime}$ are the corresponding anisotropic anomalous dimensions and $r=\alpha_{O}^{\prime} / \alpha_{P}^{\prime}$ is the ratio between the slopes. One immediately notes that $\lambda_{3}=0$ so that the critical theory has no operator changing the number of Odderon pairs. Apart from the anomalous dimensions, the universal critical exponents are extracted with a spectral analysis of the stability matrix, obtained perturbing the beta functions around the fixed point. We find two negative eigenvalues, associated to two relevant directions, which give the standard corresponding critical exponents $\nu$ related to the behavior of the two point functions for the pomeron and odderon:

$$
\lambda^{(1)}=-2+\frac{\epsilon}{4} \rightarrow \nu_{P}=\frac{1}{2}+\frac{\epsilon}{16} \quad, \quad \lambda^{(2)}=-2+\frac{\epsilon}{12} \rightarrow \nu_{O}=\frac{1}{2}+\frac{\epsilon}{48} .
$$

This fixed point (as well as its non perturbative counterpart for $D=2$ ) is important in order to understand the renormalization group flow properties. Indeed we expect that from the QCD description at short distance moving to larger and larger transverse distances one gets closer to an effective RFT formulation. As a last remark we can add that this system is in the same universality class of an out of equilibrium directed percolating system, as was shown to be the case by J. Cardy for a purely single pomeron RFT. 


\subsection{The running coupling constant}

\subsubsection{Lipatov's proposal}

The perturbative QCD pomeron and odderon in the LLA come from a summation of terms of the order $\left(\alpha_{s} \ln s\right)^{n}$ but neglect terms of the order $\left(\alpha_{s} \ln k^{2}\right)^{n}$ responsible for the running of the coupling. So in this theory $\alpha_{s}$ is assumed to be a fixed (small) parameter. As a result the BFKL theory neglects one of the basic properties of the QCD - the running of the coupling and so the asymptotic freedom. It also automatically neglects its other basic properties - the confinement, which manifests itself in the growth of the coupling at small $k^{2}$. It is then natural that nearly immediately after the introduction of the BFKL pomeron attempts to introduce the running of the coupling in the theory appeared .

The first of these attempts was made by L.N. Lipatov as early as in 1986 [40]. It was rather straightforward. Let the BFKL Hamiltonian $H$ be written with the separated factor $\alpha_{s}$, namely $H=\alpha_{s} h$. Consider the forward case, so that the BFKL equation is given by

$$
\alpha_{s}(h \psi)(k)=E \psi(k), \quad \text { or }(h \psi)(k)=\chi \psi(k)
$$

with the set of eigenvalues $\chi_{\nu, n}$ and eigenfunctions $\psi_{\nu, n}$

$$
\chi_{\nu, n}=2 \hat{\psi}(1)-\hat{\psi}\left(\frac{1}{2}+i \nu\right)-\hat{\psi}\left(\frac{1}{2}-i \nu\right), \quad \psi_{\nu, n}(k)=c k^{2 i \nu-1 / 2} e^{i n \phi},
$$

where $\hat{\psi}$ is the logarithmic derivative of the $\Gamma$-function, and $-\infty<\nu<+\infty$, $n=0, \pm 1, \pm 2, \ldots$.

The idea of L.N. Lipatov was to change the fixed $\alpha_{s}$ in (1.37) into the running one as a function of $k$

$$
\alpha_{s} \rightarrow \frac{1}{\beta_{0} \ln \left(k^{2} / \Lambda_{Q C D}^{2}\right)}, \quad \beta_{0}=\frac{11 N_{c}-2 N_{f}}{12 \pi}=\frac{b}{\pi} .
$$

Rescaling $k \rightarrow k / \Lambda_{Q C D}$ Eq. 1.37 then turns into

$$
(h \psi)(k)=\omega \beta_{0} \ln \left(k^{2}\right) \psi(k) .
$$

Now one passes to the $(\nu, n)$ representation

$$
\psi(k)=\int d \nu \sum_{n} \psi_{\nu, n} a_{\nu, n}
$$

and obtains an equation for $a_{n, n u}$

$$
i \omega \beta_{0} \frac{d a_{\nu, n}}{d \nu}=\chi(\nu, n) a_{\nu, n}
$$

which evidently admits an explicit solution.

The next step is to impose the appropriate boundary conditions. Addressing the reader to the original paper for details, we only mention that they are two, following from the behavior of the resulting $\psi(k)$ at large and small $k$. At large $k$ it is required that the solution pass to the known form for the DGLAP equation in 
the double log approximation. At small $k$ it is required that the phase of $\psi$ should be fixed from the non-perturbative region. The two boundary conditions make the spectrum a discrete one, which for the $n$-th state with $n>>1$ is

$$
\omega_{n}=-\frac{0.4085}{n-\frac{1}{4}+\frac{\phi_{0}-\nu t_{0}}{\pi}},
$$

where $\phi_{0}$ is the non-perturbative phase and small $t_{0}=k_{0}^{2} / \Lambda_{Q C D}^{2}$ is the point at which the low boundary condition is imposed. As a result one finds an infinite sequence of poles in the $j$ plane converging to the point $j=1$.

This way of introduction of the running coupling raises questions. Operator $h$ is in fact an integral one with a kernel $h\left(k, k^{\prime}\right)$. Change 1.39$)$ implies that one argument is used for the running. Such a choice, apart from being arbitrary, violates the intrinsic symmetry between $k$ and $k^{\prime}$ essential for the interchange projectile $\leftrightarrow$ target. The form of the boundary at small $k$ does not seem to be unique. Still the conclusion of the splitting of the cut into a series of poles as a result of the running of the coupling was later confirmed in other versions of the running coupling and so was an important prediction in this line of approach. Also the Lipatov running coupling allows to obtain many explicit results, in contrast to more elaborate versions. Later it was extensively used to obtain physically important results (the latest are $41-43]$ ). See also 44 .

\subsubsection{The running coupling constant from the bootstrap}

In search of a more fundamental basis for the introduction of the running coupling constant many years ago we turned to the bootstrap property of the BFKL dynamics, which is one of the pillars on which the idea of gluon reggeization rests [45. As is well known, the bootstrap discovered in the original papers 3, 4] is the unitarity condition for the channel with the gluon color number. It guarantees that the unitarity in this section gives rise to the solution in the form of the reggeized gluon. It was natural to expect that this condition should be preserved also with the running coupling. So preservation of the bootstrap could be the guiding principle for the introduction of the running coupling constant. Later the idea that a strong bootstrap condition in perturbation theory up to next-to-leading order was propsed in 46. 47, where non trivial relations for the NLO BFKL in the octet channel, the reggeized gluon trajectory and any inpact factor were conjectured. They were later on veryfied by V. Fadin and collaborators 48 .

To realize this idea one can write the inhomogeneous equation for two reggeons as

$$
(H-E) \psi=F,
$$

with the Hamiltonian for the color group $S U\left(N_{c}\right)$

$$
H=-\omega\left(q_{1}\right)-\omega\left(q_{2}\right)+\left(T_{1} T_{2}\right) V .
$$


The form of $\omega(q)$ and interaction $V$ are in fact interrelated. To make it explicit we express both via a single function $\eta(q)$ 1 ${ }^{1}$ Namely

$$
\omega(q)=\frac{1}{2} N_{c} \int \frac{d^{2} q_{1}}{(2 \pi)^{2}} \frac{\eta(q)}{\eta\left(q_{1}\right) \eta\left(q-q_{1}\right)} .
$$

The interaction term, apart from the product of the gluon color vectors $T_{i}^{a}, i=$ $1,2, a=1, \ldots N_{c}$, involves the interaction kernel $V$ which we also express via the same function $\eta(q)$

$$
V\left(q_{1}, q_{2} \mid q_{1}^{\prime} q_{2}^{\prime}\right)=\frac{\eta\left(q_{1}+q_{2}\right)}{\eta\left(q_{1}^{\prime}\right) \eta\left(q_{2}^{\prime}\right)}-\left(\frac{\eta\left(q_{1}\right)}{\eta\left(q_{1}^{\prime}\right)}+\frac{\eta\left(q_{2}\right)}{\eta\left(q_{2}^{\prime}\right)}\right) \frac{1}{\eta\left(q_{1}-q_{1}^{\prime}\right)} .
$$

It conserves the momentum: $q_{1}+q_{2}=q_{1}^{\prime}+q_{2}^{\prime}$. Combining (1.46) with 1.47) for the gluon channel with $\left(T_{1} T_{2}\right)=-\frac{1}{2} N_{c}$ one finds the bootstrap relation for any $\eta(q)$ :

$$
\frac{1}{2} N_{c} \int \frac{d^{2} q_{1}}{(2 \pi)^{2}} V\left(q_{1}, q_{2} \mid q_{1}^{\prime}, q_{2}^{\prime}\right)=\omega\left(q_{1}\right)+\omega\left(q_{2}\right)-\omega\left(q_{1}+q_{2}\right) .
$$

Then taking in the inhomogeneous equation $F=F\left(q_{1}+q_{2}\right)$ one finds a solution to Eq. (1.44):

$$
\psi\left(q_{1}, q_{2}\right)=\psi\left(q_{1}+q_{2}\right)=\frac{F\left(q_{1}+q_{2}\right)}{\omega\left(q_{1}+q_{2}\right)-E} .
$$

This means that the two gluons 1 and 2 have fused into a single one with the momentum $q_{1}+q_{2}$ and the moving pole at $j=1-E$. This phenomenon is true for arbitrary $\eta(q)$.

For the original BFKL equation with the fixed coupling we have a particular $\eta(q):$

$$
\eta(q)=\frac{2 \pi}{g^{2}} q^{2}, \quad \eta(0)=0 .
$$

Then one finds the standard LL BFKL expressions for the trajectory $\omega(q)$ and interaction $V\left(q_{1}, q_{2} \mid q_{1}^{\prime}, q_{2}^{\prime}\right)$. Also the trajectory passes zero at $q=0$ to correspond to the real gluon with a vanishing mass.

Using the fact that the bootstrap is fulfilled for arbitrary $\eta(q)$ we can choose $\eta(q)$ to satisfy the high-momentum behavior of the gluon distribution with a running coupling 45

$$
\eta(q)=\frac{b}{2 \pi} q^{2} \ln \frac{q^{2}}{\Lambda_{Q C D}^{2}}, \quad q^{2}>>\Lambda^{2},
$$

where $\Lambda$ is the standard QCD parameter and $b=(11 N c-2 N f) / 12$ is defined in 1.39 .

The asymptotic (1.51) and condition $\eta(0)=0$ are the only properties of $\eta(q)$ which follow from the theoretical reasoning. A concrete form of $\eta(q)$ interpolating between $q^{2}=0$ and 1.51 may be chosen differently. One hopes that physical results will not too strongly depend on the choice.

\footnotetext{
${ }^{1}$ We stress that this is just a possible way, but not the unique one, to satisfy the bootstrap relation.
} 
With $\eta(q)$ satisfying 1.51 we can build the trajectory $\omega(q)$ and the interaction $V$ between two reggeons with the running coupling constant. In this way we obtain equations for both pomeron, odderon and higher BKP states with the running coupling constant. Note that as with the fixed coupling the resulting equations remain infrared stable if $\eta(0)=0$ as dictated by the zero gluon mass. However we can improve the infrared behavior to mimic confinement and introduce a finite gluon mass $m$ dropping the latter condition. In our practical applications we use

$$
\eta(q)=\frac{b}{2 \pi} f(q), \quad f(q)=\left(q^{2}+m^{2}\right) \ln \frac{q^{2}+m^{2}}{\Lambda^{2}} .
$$

With this choice $\eta(0) \neq 0$ and has to be taken into account in the interaction.

It is interesting to note that this very simple ansatz given in Eqs. (1.46) and 1.47 in order to satisfy the bootstrap condition, when feeded with the choice of Eq. 1.51 can be studied to compare to the results of the NLL BFKL kernel and the external particle impact factors. This can be conveniently done using dimensional regularization [47] where it was shown that fermionic contributions (the $N_{f}$ dependent part) was correctly reproduced, as well as the gluon sector related to the running coupling. Only a gluon contribution of the NLL approximation is actually missing. This is therefore an interesting approximation containing an important part of the NLL corrections and can be considered as a good starting point to study the running coupling effects.

\subsubsection{The BLV odderon with the running coupling constant}

For the odderon the color wave function is $d_{a_{1} a_{2} a_{3}}$ where $a_{i}$ is the color of the $i$-th reggeon. Separating it, the equation for the odderon momentum wave function is

$$
\left(H_{o d}-E\right) \psi_{o d}=0,
$$

with a Hamiltonian

$$
H_{o d}=-\sum_{i=1}^{3} \omega\left(q_{i}\right)-\frac{1}{2} N_{c} \sum_{i<k}^{3} V_{i k} .
$$

Consider the odderon equation with a specific inhomogeneous term

$$
\left(H_{o d}-E\right) \psi_{12}=F_{12},
$$

where

$$
\left.F_{12}(1,2,3)=\int \frac{d^{2} q_{3}^{\prime}}{(2 \pi)^{2}} \hat{W}\left(1,2,3 \mid 1^{\prime}, 3^{\prime}\right)\right) \psi\left(1^{\prime}, 3^{\prime}\right)+F_{2}(1,2,3),
$$

where for brevity we denote momenta $q_{1}, q_{2}, q_{3}$ just by their numbers $1,2,3$ and so on. Here $q_{1}^{\prime}+q_{3}^{\prime}=q_{1}+q_{2}+q_{3}$. In Eq. 1.56 $\psi\left(1^{\prime}, 3^{\prime}\right)$ is some function of two momenta $q_{1}^{\prime}$ and $q_{3}^{\prime}$. The kernel $\hat{W}$ is a sum of two terms

$$
\hat{W}\left(1,2,3 \mid 1^{\prime}, 3^{\prime}\right)=-\frac{1}{2} N_{c}\left(W\left(2,1,3 \mid 1^{\prime}, 3^{\prime}\right)+W\left(1,2,3 \mid 1^{\prime}, 3^{\prime}\right)\right)
$$


and $W$ is a difference between two kernels (1.47), expressed via function $\eta(q)$. In full notation

$$
W\left(q_{2}, q_{1}, q_{3} \mid q_{1}^{\prime}, q_{3}^{\prime}\right)=V\left(q_{1}, q_{3} \mid q_{1}^{\prime}-q_{2}, q_{3}^{\prime}\right)-V\left(q_{1}+q_{2}, q_{3} \mid q_{1}^{\prime}, q_{i}^{\prime}\right) .
$$

It possesses an important symmetry property:

$$
W\left(1,2,3 \mid 1^{\prime}, 3^{\prime}\right)=W\left(3,2,1 \mid 3^{\prime}, 1^{\prime}\right) .
$$

Note that in the fixed coupling case this kernel reduces to the Bartels kernel $K_{2 \rightarrow 3}$ which describes transition from two BFKL pomerons to three [23]. Therefore one could be tempted to introduce in a similar way the running coupling also in the effective vertices which change the number of ( $t$-channel) reggeized gluons, and in particular the pomeron $\rightarrow 2$ pomeron and the pomeron $\rightarrow 2$ odderon vertices whose expressions are known at fixed coupling [7, 23].

It can be shown [49] that the inhomogeneous equation (1.55) can be solved by

$$
\psi_{12}\left(q_{1}, q_{2}, q_{3}\right)=\psi\left(q_{1}+q_{2}, q_{3}\right)
$$

provided function $\psi$ is a pomeron wave function which satisfies the homogeneous Schroedinger equation (1.44) with a Hamiltonian 1.45 in which $T_{1} T_{2}=-N_{c}$ (the BFKL equation in the case of a fixed coupling).

Using this, we cyclically permute the gluons $1,2,3$ to obtain two more relations

$$
\left(H_{o d}-E\right) \psi_{23}=F_{23}
$$

and

$$
\left(H_{o d}-E\right) \psi_{31}=F_{31},
$$

where for instance

$$
\psi_{23}\left(q_{1}, q_{2}, q_{3}\right)=\psi\left(q_{2}+q_{3}, q_{1}\right)
$$

and

$$
F_{23}(1,2,3)=-\frac{1}{2} N_{c} \int \frac{d^{2} q_{3}^{\prime}}{(2 \pi)^{2}}\left(W\left(3,2,1 \mid 1^{\prime}, 3^{\prime}\right)+W\left(2,3,1 \mid 1^{\prime}, 3^{\prime}\right)\right) \psi\left(1^{\prime}, 3^{\prime}\right)
$$

(the integration momenta $q_{3}^{\prime}$ and $q_{1}^{\prime}=q_{1}+q_{2}+q_{3}-q_{3}^{\prime}$ do not change under permutations of the external momenta). We add all the three equations obtained in this manner together to obtain

$$
\left(H_{o d}-E\right)\left(\psi_{12}+\psi_{23}+\psi_{31}\right)=F_{12}+F_{23}+F_{31} \equiv F_{t o t} .
$$

The total inhomogeneous term is

$$
F_{\text {tot }}(1,2,3)=-\frac{N_{c}}{2} \int \frac{d^{2} q_{3}^{\prime}}{(2 \pi)^{2}} U\left(1,2,3 \mid 1^{\prime}, 3^{\prime}\right) \psi\left(1^{\prime}, 3^{\prime}\right)
$$

where

$$
\begin{gathered}
U\left(1,2,3 \mid 1^{\prime}, 3^{\prime}\right)=W\left(2,1,3 \mid 1^{\prime}, 3^{\prime}\right)+W\left(1,2,3 \mid 1^{\prime}, 3^{\prime}\right)+W\left(3,2,1 \mid 1^{\prime}, 3^{\prime}\right) \\
+W\left(2,3,1 \mid 1^{\prime}, 3^{\prime}\right)+W\left(1,3,2 \mid 1^{\prime}, 3^{\prime}\right)+W\left(3,1,2 \mid 1^{\prime}, 3^{\prime}\right) .
\end{gathered}
$$


Using property 1.59 one easily finds that $U$ is symmetric in the last pair of arguments

$$
U\left(1,2,3 \mid 1^{\prime}, 3^{\prime}\right)=U\left(1,2,3 \mid 3^{\prime}, 1^{\prime}\right) .
$$

It follows that if function $\psi\left(q_{1}, q_{2}\right)$ is antisymmetric in its variables, the total inhomogeneous term 1.66 in Eq. 11.65 vanishes and the sum

$$
\psi_{\text {od }}=\psi_{12}+\psi_{23}+\psi_{31}
$$

is a solution of the odderon equation

$$
\left(H_{o d}-E\right) \psi_{o d}=0 .
$$

Thus from any antisymmetric solution of the pomeron equation one gets a corresponding solution of the odderon equation.

For a fixed coupling this result was found in [19]. Our derivation generalizes it to the case of a running coupling, provided it is introduced in the manner which preserves the bootstrap.

\subsubsection{A general family of BKP states in the planar limit with the running coupling constant}

We consider here an $n$ gluon composite state in color singlet which has the high energy behavior in the regge limit described by the following kernel

$$
H_{n}=-\sum_{i=1}^{n} \omega\left(q_{i}\right)+\sum_{i<j} T_{i} T_{j} V_{i j}
$$

which in the planar limit reduces to

$$
H_{n}^{\infty}=\frac{1}{2}\left[H_{12}^{(1)}+H_{23}^{(1)}+\cdots+H_{n 1}^{(1)}\right]
$$

and where $H_{i j}$ is a two gluon Hamiltonian acting on the gluons $i$ and $j$. As for the case of the previous section we shall proceed with the replacement from the fixed coupling case of Eq. 1.50 to the running coupling one of Eq. 1.51, this time following an approach similar to [50]. Starting from the ansatz

$$
\psi_{n}\left(k_{1}, k_{2}, \cdots, k_{n}\right)=\sum_{i=0}^{n-1}\left(R_{n}\right)^{i} c_{i} \psi_{n-1}\left(k_{1}+k_{2}, k_{3}, \cdots, k_{n}\right),
$$

where $R_{n}$ is the generator of a cyclic shift along the cylinder, i.e. $\left(R_{n}\right)^{n}=1$ and $c_{i}$ are constant to be determined, and rearranging the Hamiltonian in the form

$$
\bar{H}_{n}^{\infty}(1,2, \cdots, n)=\frac{1}{2}\left(\bar{H}_{12}^{(1)}+\bar{H}_{1 n}^{(1)}-\bar{H}_{2 n}^{(1)}\right)+\bar{H}_{n-1}^{\infty}(2,3, \cdots, n),
$$

let us consider the action of the Hamiltonian on the function $g=\psi_{n-1}\left(k_{1}+\right.$ $\left.k_{2}, k_{3}, \cdots, k_{n}\right)$. One finds

$$
\begin{aligned}
& \bar{H}_{n}^{\infty}(1,2, \cdots, n) \otimes g= \\
& \int\left\{d^{2} k_{i}^{\prime}\right\} \bar{H}_{n-1}^{\infty}\left(k_{1}+k_{2}, k_{3}, \cdots, k_{n} \mid k_{2}^{\prime}, k_{3}^{\prime}, \cdots, k_{n}^{\prime}\right) \psi_{n-1}\left(k_{2}^{\prime}, k_{3}^{\prime}, \cdots, k_{n}^{\prime}\right)+ \\
& \frac{1}{2} \int\left\{d^{2} k_{i}^{\prime}\right\} W\left(k_{1}, k_{2}, k_{3} \mid k_{1}^{\prime}, k_{3}^{\prime}\right) \psi_{n-1}\left(k_{1}^{\prime}, k_{3}^{\prime}, \cdots, k_{n}^{\prime}\right)+ \\
& \frac{1}{2} \int\left\{d^{2} k_{i}^{\prime}\right\} W\left(k_{2}, k_{1}, k_{n} \mid k_{2}^{\prime}, k_{n}^{\prime}\right) \psi_{n-1}\left(k_{2}^{\prime}, k_{3}^{\prime}, \cdots, k_{n}^{\prime}\right),
\end{aligned}
$$


where we denote with $\left\{d^{2} k_{i}^{\prime}\right\}$ the necessary integral measure. Imposing for $\psi_{n-1}$ the following properties: (1) it is an eigenstate, i.e. $\bar{K}_{n-1}^{\infty} \psi_{n-1}=E \psi_{n-1}$ and (2) has definite symmetry such that $R_{n-1} \psi_{n-1}=r_{n-1} \psi_{n-1}$, one can write the previous relation as

$$
\begin{aligned}
& \bar{H}_{n}^{\infty}(1,2, \cdots, n) \otimes \psi_{n-1}\left(k_{1}+k_{2}, k_{3}, \cdots, k_{n}\right)=E \psi_{n-1}\left(k_{1}+k_{2}, k_{3}, \cdots, k_{n}\right)+ \\
& \frac{1+r_{n-1}\left(R_{n}\right)^{-1}}{2} \int\left\{d^{2} k_{i}^{\prime}\right\} W\left(k_{1}, k_{2}, k_{3} \mid k_{1}^{\prime}, k_{3}^{\prime}\right) \psi_{n-1}\left(k_{1}^{\prime}, k_{3}^{\prime}, \cdots, k_{n}^{\prime}\right)
\end{aligned}
$$

so that, using the definition of Eq. 1.73 we can have the relation

$$
\bar{H}_{n}^{\infty} \psi_{n}=E \psi_{n}
$$

provided the unknown coefficients $c_{i}$ satisfy the secular equation

$$
\sum_{i=0}^{n-1}\left(R_{n}\right)^{i}\left(1+r_{n-1}\left(R_{n}\right)^{-1}\right) c_{i}=0
$$

where $r_{n-1}$ plays the role of eigenvalue and $c_{i}$ are the components of the corresponding eigenvector. Using the constraint $\left(r_{n-1}\right)^{n-1}=1$, one finds $r_{n-1}=(-1)^{n}$. Therefore for $n$ even one has $r_{n-1}=+1$ and $c_{i}=(-1)^{i}$ while for $n$ odd we get $r_{n-1}=+1$ and $c_{i}=+1$. The case with $n$ even is unphysical because the state $\psi_{n}$ would be not Bose symmetric. Instead the case with $n$ odd is physical, with the solution constructed from another solution for $n-1$ gluons which is odd under cyclic permutations. In particular the odderon corresponds to the special case $n=3$. In summary, given for an even number of gluons a solution of the kernel, improved to include running coupling effects using the bootstrap approach, with some intercept eigenvalue, then one can automatically construct another solution with one more gluon with the same intercept.

\subsubsection{Equations for the pomeron and BLV odderon with the run- ning coupling}

The BFKL equation admits explicit solutions both in the forward and non-forward directions based on its conformal invariance. With the running coupling this invariance is lost and one has to relay on numerical studies. The first calculations of the pomeron ground states intercept and slope were done nearly immediately after the introduction of the running coupling from the bootstrap [51. Here we expand these calculations and extend them to the BLV odderon. We borrow from 51 the starting equations in the form suitable for numerical calculations.

We put $N_{c}=3$, present $\eta(q)$ according to 1.52 and remove all numerical factors including $b$ from the Hamiltonian redefining the eigenvalue. We also symmetrize the integral kernel in $V$ by introducing a new wave function $\phi$ by

$$
\psi\left(q_{1}\right)=\phi\left(q_{1}\right) \sqrt{f\left(q_{1}\right) f\left(q_{2}\right)}, \quad q_{1}+q_{2}=q
$$

where $q$ is the total momentum. Then the equation for $\phi$ takes the form

$$
\int d^{2} q_{1}^{\prime} h_{q}\left(q_{1}, q_{1}^{\prime}\right) \phi\left(q_{1}^{\prime}\right)=A_{q}\left(q_{1}\right) \phi\left(q_{1}\right)+\int d^{2} q_{1}^{\prime} L_{q}\left(q_{1}, q_{1}^{\prime}\right) \phi\left(q_{1}^{\prime}\right)=\epsilon(q) \phi\left(q_{1}\right)
$$


Here the "kinetic energy" is

$$
A_{q}\left(q_{1}\right)=(1 / 2) \int \frac{d^{2} q_{1}^{\prime} f\left(q_{1}\right)}{f\left(q_{1}^{\prime}\right) f\left(q_{1}-q_{1}^{\prime}\right)}+(1 / 2) \int \frac{d^{2} q_{2}^{\prime} f\left(q_{2}\right)}{f\left(q_{2}^{\prime}\right) f\left(q_{2}-q_{2}^{\prime}\right)} .
$$

The interaction kernel consists of two parts, a quasilocal and a separable ones:

$$
L=L^{(q l)}+L^{(s e p)}
$$

They are given by

$$
\left.L_{q}^{(q l)}\left(q_{1}, q_{1}^{\prime}\right)=-\sqrt{\frac{f\left(q_{1}\right)}{f\left(q_{2}\right)}} \frac{1}{f\left(q_{1}-q_{1}^{\prime}\right)} \sqrt{\frac{f\left(q_{2}^{\prime}\right)}{f\left(q_{1}^{\prime}\right)}}-\sqrt{\frac{f\left(q_{2}\right)}{f\left(q_{1}\right)}} \frac{1}{f\left(q_{2}-q_{2}^{\prime}\right)} \sqrt{\frac{f\left(q_{1}^{\prime}\right)}{f\left(q_{2}^{\prime}\right)}}\right)
$$

and

$$
L_{q}^{(s e p)}\left(q_{1}, q_{1}^{\prime}\right)=\frac{f(q)}{\sqrt{f\left(q_{1}\right) f\left(q_{2}\right) f\left(q_{1}^{\prime}\right) f\left(q_{2}^{\prime}\right)}} .
$$

Both parts are evidently symmetric in $q_{1}$ and $q_{1}^{\prime}$. The scaled energy $\epsilon$ is related to the initial one by

$$
E=\frac{3}{2 \pi b} \epsilon=\frac{6}{\pi\left(11-(2 / 3) N_{F}\right)} \epsilon .
$$

Solutions of Eq. 1.80 give the pomeron energies and wave function. Its solutions antisymmetric in $q_{1} \leftrightarrow q_{2}$ give energies and wave functions of the BLV odderon.

The nonforward equation 1.80 with $q \neq 0$ is too complicated for the numerical study due to three independent variables. So we concentrate on the forward case $q=0$.

At $q=0$ the equation retains its form 1.80 with

$$
A_{0}\left(q_{1}\right)=\int \frac{d^{2} q_{1}^{\prime} f\left(q_{1}\right)}{f\left(q_{1}^{\prime}\right) f\left(q_{1}-q_{1}^{\prime}\right)}
$$

and the interaction given by (17) where now

$$
L_{0}^{(q l)}\left(q_{1}, q_{1}^{\prime}\right)=-\frac{2}{f\left(q_{1}-q_{1}^{\prime}\right)}
$$

has really become local and

$$
L_{0}^{(s e p)}\left(q_{1}, q_{1}^{\prime}\right)=\frac{f(0)}{f\left(q_{1}\right) f\left(q_{1}^{\prime}\right)} .
$$

In the following we omit the subindex 0 implying $q=0$.

To keep under control the behavior of the kernel at extremely large values of momenta we transform the trajectory term $A(q)$ similar to what is standardly done in the BFKL equation. Using the identity

$$
\int d^{2} q_{1} \frac{f(q)}{f\left(q_{1}\right) f\left(q-q_{1}\right)}=2 \int d^{2} q_{1} \frac{f(q)}{f\left(q-q_{1}\right)\left(f\left(q_{1}\right)+f\left(q-q_{1}\right)\right)},
$$


we find

$$
A(q)=\int d q_{1}^{2} \int_{0}^{2 \pi} d \chi_{1} \frac{f(q)}{f\left(q-q_{1}\right)\left(f\left(q_{1}\right)+f\left(q-q_{1}\right)\right)} .
$$

where $\chi_{1}$ is the azimuthal angle of $q_{1}$.

To make the equation for $\phi$ one-dimensional, the angular momentum $n$ of the gluons is introduced

$$
\phi(q)=\phi_{n}\left(q^{2}\right) e^{i n \chi},
$$

where $\chi$ is the azimuthal angle. Integrating over it in the equation, one obtains an one-dimensional integral equation for the radial function $\phi_{n}\left(q^{2}\right)$ :

$$
A(q) \phi_{n}\left(q^{2}\right)+\int d q_{1}^{2} L_{n}\left(q^{2}, q_{1}^{2}\right) \phi_{n}\left(q_{1}^{2}\right)=\epsilon \phi_{n}\left(q^{2}\right),
$$

with the kernel $L_{n}$ now given by

$$
L_{n}\left(q^{2}, q_{1}^{2}\right)=-B_{n}\left(q^{2}, q_{1}^{2}\right)+\delta_{n 0} \pi \frac{f(0)}{f(q) f\left(q_{1}\right)},
$$

where

$$
B_{n}\left(q^{2}, q_{1}^{2}\right)=\int_{0}^{2 \pi} d \chi \frac{\cos n \chi}{f\left(q^{2}+q_{1}^{2}-2 q q_{1} \cos \chi\right)} .
$$

The kinetic term $A(q)$ after the angular integration in Eq. 1.89 can be written as

where

$$
A(q)=\int d q_{1}^{2} \frac{f(q)}{f\left(q_{1}\right)}\left(B_{0}\left(q, q_{1}\right)-C_{0}\left(q, q_{1}\right)\right),
$$

$$
C_{0}\left(q, q_{1}\right)=\int_{0}^{2 \pi} d \chi \frac{1}{f\left(q_{1}\right)+f\left(q-q_{1}\right)} .
$$

These are the equations we are solving numerically. We shall see that the ground state of the pomeron with negative energies indeed splits into an infinite series of discrete states, with energies converging to $E=0$. In contrast the odderon states remain continuous with energies along a cut in the $j$ plane starting from $j=1$. As a result we expect that in the non-forward direction the discrete pomeron states will move with the total momentum $q$ with the intercept $\alpha(q)$. As to the continuous odderon states we expect them to stay on the cut above $E=0$. Should they move, it may introduce moving unphysical cuts in the $t$-plane and violate the unitarity.

As mentioned the equations become very difficult to solve at $q \neq 0$ even numerically. So, rather than to attempt to solve them for all $q$, we limit ourselves to small values of $q$ and determine not the whole trajectory $\alpha(q)$ but only the intercept $\alpha^{\prime}$ defined by

$$
\alpha(q)=1-E(q)=1+\Delta-\alpha^{\prime}(0) q^{2} .
$$

This can be done in a much simpler manner using a perturbative approach. We present "the Hamiltonian" in 1.80

$$
H_{q}=A_{q}+L_{q}=H_{0}+W(q)
$$


and calculate analytically $W(q)$ up to terms of the second order in $q$. Then for small $q$ the value of the energy $\epsilon(q)$ will be given by the standard perturbation formula

$$
\epsilon(q)=\epsilon(0)+\langle W(q)\rangle,
$$

where \langle\rangle here means taking the average with the wave function at $q=0$, determined from the numerical solution of the equation discussed above. Note that for the continuous and non-normalizable states $\langle W(q)\rangle=0$ is expected unless the interaction is too bad. Our numerical studies confirm this result.

Technically it is natural to write the momenta as

$$
q_{1}=\frac{1}{2} q+l, \quad q_{2}=\frac{1}{2} q-l, \quad q_{1}^{\prime}=\frac{1}{2} q+l^{\prime}, \quad q_{2}^{\prime}=\frac{1}{2} q-l^{\prime}
$$

and expand in power series all expressions in 1.80 up to the second order in $q$. The corresponding calculations are straightforward but tedious and so together with the result they are briefly presented in the Appendix.

\subsubsection{Change of variable and discretization}

We pass to the logarithmic variable $t=\ln q^{2}$ (the unit in which $q^{2}$ is measured is inferred from the numerical values of the dimensional parameters $m$ and $\Lambda_{Q C D}$ ). To retain the symmetry of the kernel we express $\psi(q)$ as

$$
\phi_{n}\left(q^{2}\right)=\tilde{\phi}_{n}\left(q^{2}\right) / q .
$$

The equation becomes

$$
A(q) \tilde{\phi}_{n}\left(q^{2}\right)+\int d t_{1} q q_{1} L_{n}\left(q^{2}, q_{1}^{2}\right) \tilde{\phi}_{n}\left(q_{1}^{2}\right)=\epsilon \tilde{\phi}_{n}\left(q^{2}\right),
$$

where $q^{2}=e^{t}$ and $q_{1}^{2}=e_{1}^{t}$. Here

$$
A(q)=\int d t_{1} q_{1}^{2} \frac{f(q)}{f\left(q_{1}\right)}\left(B_{0}\left(q^{2}, q_{1}^{2}\right)-C_{0}\left(q^{2}, q_{1}^{2}\right)\right) .
$$

The discretization is realized using a uniform grid in $t$

$$
t_{i}=t_{\min }+i d, \quad i=0,1, \cdots, N, \quad d=\frac{t_{\max }-t_{\min }}{N} .
$$

We approximate the integrals over $t$ by finite sums

$$
\int_{-\infty}^{\infty} d t F(t) \simeq \sum_{i=0}^{N} w_{i} F\left(t_{i}\right)
$$

with points $t_{i}$ and weights $w_{i}$ depending on the chosen approximation scheme.

Then Eq. 1.101 becomes a finite linear system of equations, where the kernel has become a matrix. To symmetrize it we finally introduce

$$
\tilde{\phi}_{i}=\frac{v_{i}}{\sqrt{w_{i}}}, \quad \text { or } \phi\left(q_{i}^{2}\right)=\frac{1}{\sqrt{q_{i}^{2} w_{i}}} v_{i}
$$


and the final equation is

$$
A_{i} v_{i}+\sum_{j=0}^{N} \sqrt{w_{i} w_{j}} q_{i} q_{j} L_{n}\left(q_{i}^{2}, q_{j}^{2}\right) v_{j}=\epsilon v_{i}
$$

with

$$
A_{i}=\sum_{j=0}^{N} w_{j} q_{j}^{2} \frac{f\left(q_{i}\right)}{f\left(q_{j}\right)}\left(B_{0}\left(q_{i}^{2}, q_{j}^{2}\right)-C_{0}\left(q_{i}^{2}, q_{j}^{2}\right)\right) .
$$

We are going to find the eigenvalues and eigenvectors of the matrix associated to the linear operator of the equation. The matrix is

$$
M_{i j}=A_{i} \delta_{i j}+\sqrt{w_{i} w_{j}} q_{i} q_{j}\left(-B_{n}\left(q_{i}^{2}, q_{j}^{2}\right)+\delta_{n 0} \pi \frac{f(0)}{f\left(q_{i}\right) f\left(q_{j}\right)}\right) .
$$

Its diagonal term is

$$
M_{i i}=A_{i}+w_{i} q_{i}^{2}\left(-B_{n}\left(q_{i}^{2}, q_{i}^{2}\right)+\delta_{n 0} \pi \frac{f(0)}{f^{2}\left(q_{i}\right)}\right) .
$$

In order to make explicit the behavior at large momentum, i.e. at large $t$, we separate in $A_{i}$ from the sum over $j$ the term $j=i$ and write

$$
A_{i}=w_{i} q_{i}^{2}\left(B_{0}\left(q_{i}^{2}, q_{i}^{2}\right)-C_{0}\left(q_{i}^{2}, q_{i}^{2}\right)\right)+S,
$$

where

$$
S=\sum_{j=0, j \neq i} w_{j} q_{j}^{2} \frac{f\left(q_{i}\right)}{f\left(q_{j}\right)}\left(B_{0}\left(q_{i}^{2}, q_{j}^{2}\right)-C_{0}\left(q_{i}^{2}, q_{j}^{2}\right)\right),
$$

and combine the separated term with the second term in 1.109$)$ to obtain

$$
M_{i i}=w_{i} q_{i}^{2}\left(B_{0}\left(q_{i}^{2}, q_{i}^{2}\right)-B_{n}\left(q_{i}^{2}, q_{i}^{2}\right)-C_{0}\left(q_{i}^{2}, q_{i}^{2}\right)+\delta_{n 0} \pi \frac{f(0)}{f\left(q_{i}\right)^{2}}\right)+S .
$$

Here the extra large term at large $q_{i}$ is canceled between $B_{0}\left(q_{i}^{2}, q_{i}^{2}\right)$ and $B_{n}\left(q_{i}^{2}, q_{i}^{2}\right)$. So denoting

we find finally

$$
B_{0 n}\left(q_{i}^{2}\right)=B_{0}\left(q_{i}^{2}, q_{i}^{2}\right)-B_{n}\left(q_{i}^{2}, q_{i}^{2}\right)
$$

$$
M_{i i}=w_{i} q_{i}^{2}\left(B_{0 n}\left(q_{i}^{2}\right)-C_{0}\left(q_{i}^{2}, q_{i}^{2}\right)+\delta_{n 0} \pi \frac{f(0)}{f\left(q_{i}\right)^{2}}\right)+S .
$$

In this form calculation of eigenvalues of $M$ can be implemented numerically with not much efforts 2

Note that for the pomeron with $n=0$ the first term in 1.114 is zero and we get

$$
M_{i i}^{n=0}=w_{i} q_{i}^{2}\left(-C_{0}\left(q_{i}^{2}, q_{i}^{2}\right)+\pi \frac{f(0)}{f\left(q_{i}\right)^{2}}\right)+S .
$$

For the odderon $(\mathrm{n}=1)$ the last term in (1.114) is zero and one has

$$
M_{i i}^{n=1}=w_{i} q_{i}^{2}\left(B_{01}\left(q_{i}^{2}\right)-C_{0}\left(q_{i}^{2}, q_{i}^{2}\right)\right)+S .
$$

In both cases $S$ is given by 1.111 .

In this form the matrix $M$ is suitable for the determination of eigenvalues and eigenvectors with standard numerical techniques. We shall use a grid up to $t_{\max }=$ 270 .

${ }^{2}$ E.g. it can be computed with a FORTRAN program using only double precision. 


\subsection{Numerical results}

Our numerical results were obtained by the standard FORTRAN programs for finding all eigenvalues and eigenvectors of a real symmetric matrix. In the calculations made up to now we have taken

$$
m=0.82 G e V, \quad \Lambda=0.2 G e V .
$$

The lower limit in $t$ was taken in all cases as

$$
t_{\min }=-90
$$

while the upper limit was taken as

$$
t_{\max }=90,180,270 .
$$

Moreover the number of divisions $N$ was taken as

$$
N=1000 \text {, and, } 2000 \text {. }
$$

It turned out that the results practically do not change with the rise of $N$ from 1000 to 2000 , so that $N=1000$ proved to be fairly enough for a reasonable accuracy. On the other hand, some results do depend on the value of $t_{\max }$. It is worth mentioning that within the used FORTRAN program we were not able to raise it above 300 .

We considered four cases. First we repeated our old calculations for the pomeron with $n=0$ and the running coupling. Second, we considered the odderon $(\mathrm{n}=1)$ with the running coupling. Finally we studied both the pomeron and odderon with a fixed coupling regulated by the "gluon mass" $m$ in the infrared.

\subsubsection{Pomeron}

For the pomeron we have found that, in agreement with the L.N. Lipatov picture, the introduction of the running coupling splits the negative part of the cut in energy into a sequence of poles converging to the start of the cut at $E=0$. The number of negative poles rises from 19 at $t_{\max }=90$ to 55 at $t_{\max }=270$. The negative part of the spectrum at $t_{\max }=270$ is shown in Fig. 1.2. Energies and slopes of the first ten states at $t_{\max }=90$ and $t_{\max }=270$ are presented in Table 1 . One observes that their values practically do not depend on $t_{\max }$ Note that the first two energies are in agreement with our old calculations. However their slopes are not, because of a past error in the code which we have corrected. We can study the energy $\omega_{n}$ and the slope $\alpha_{n}^{\prime}$ of the state $\phi_{n}$, as a function of $n$. From a fit on the first 10 leading states we find for the "energies"

$$
\omega_{n} \simeq-\frac{0.4141}{n+0.1383}
$$

which should be compared to the behavior reported in Eq. 1.43 and which is qualitatively similar also to what found in 44 . Also the slope can be fitted. It goes 


\begin{tabular}{||c|c||c|c||}
\hline \multicolumn{2}{||c|}{$t_{\max }=90$} & \multicolumn{2}{c||}{$t_{\max }=270$} \\
\hline$-0.35582 \mathrm{E}+00$ & $0.61753 \mathrm{E}-01$ & $-0.35584 \mathrm{E}+00$ & $0.61731 \mathrm{E}-01$ \\
$-0.19280 \mathrm{E}+00$ & $0.20731 \mathrm{E}-01$ & $-0.19296 \mathrm{E}+00$ & $0.20732 \mathrm{E}-01$ \\
$-0.13205 \mathrm{E}+00$ & $0.10719 \mathrm{E}-01$ & $-0.13215 \mathrm{E}+00$ & $0.10713 \mathrm{E}-01$ \\
$-0.10025 \mathrm{E}+00$ & $0.65802 \mathrm{E}-02$ & $-0.10066 \mathrm{E}+00$ & $0.65780 \mathrm{E}-02$ \\
$-0.80711 \mathrm{E}-01$ & $0.44542 \mathrm{E}-02$ & $-0.80777 \mathrm{E}-01$ & $0.44549 \mathrm{E}-02$ \\
$-0.67512 \mathrm{E}-01$ & $0.32153 \mathrm{E}-02$ & $-0.67568 \mathrm{E}-01$ & $0.32168 \mathrm{E}-02$ \\
$-0.58005 \mathrm{E}-01$ & $0.24300 \mathrm{E}-02$ & $-0.58053 \mathrm{E}-01$ & $0.24317 \mathrm{E}-02$ \\
$-0.50834 \mathrm{E}-01$ & $0.19017 \mathrm{E}-02$ & $-0.50878 \mathrm{E}-01$ & $0.19025 \mathrm{E}-02$ \\
$-0.45214 \mathrm{E}-01$ & $0.15478 \mathrm{E}-02$ & $-0.45275 \mathrm{E}-01$ & $0.15290 \mathrm{E}-02$ \\
$-0.40496 \mathrm{E}-01$ & $0.14027 \mathrm{E}-02$ & $-0.40780 \mathrm{E}-01$ & $0.12555 \mathrm{E}-02$ \\
\hline
\end{tabular}

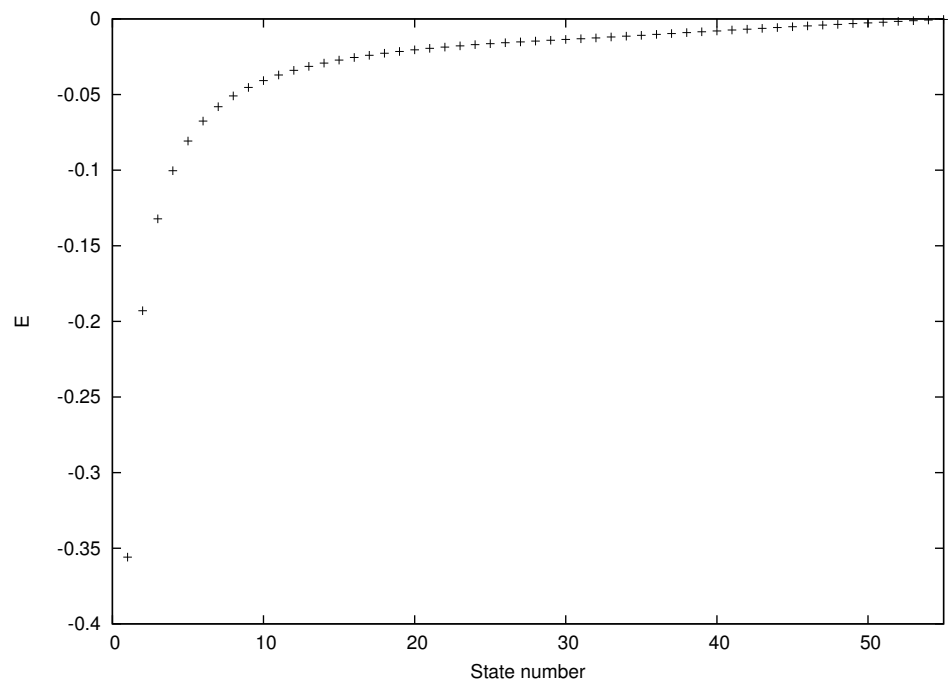

Fig. 1.2 Energies of the first 55 states of the pomeron with the running coupling. $t_{\max }=270$

roughly as $\alpha_{n}^{\prime}=a /\left(n^{2}+b\right)$ for some $a$ and $b$, even if the fit is a bit less accurate compared to what happens for the energies.

To compare we calculated energies (in units $\left|E_{B F K L}\right|$ ) and slopes for the pomeron with a fixed coupling. Here we observe a cut in energy starting at exactly the BFKL endpoint and going upwards, as predicted by previous calculation. Remarkably the number of negative states is the same as for the running coupling: 19 with $t_{\max }=90$ and 55 with $t_{\max }=270$. However the location of energies is quite different indicating a cut, as illustrated in Fig. 1.3. Also values of the intercept were found to correspond to a non-normalizable state. They are not zero but strongly diminish with the growth of $t_{\max }$, which can be seen from Table 2. Such behavior $\propto 1 / t_{\max }$ is of course expected on general grounds. 


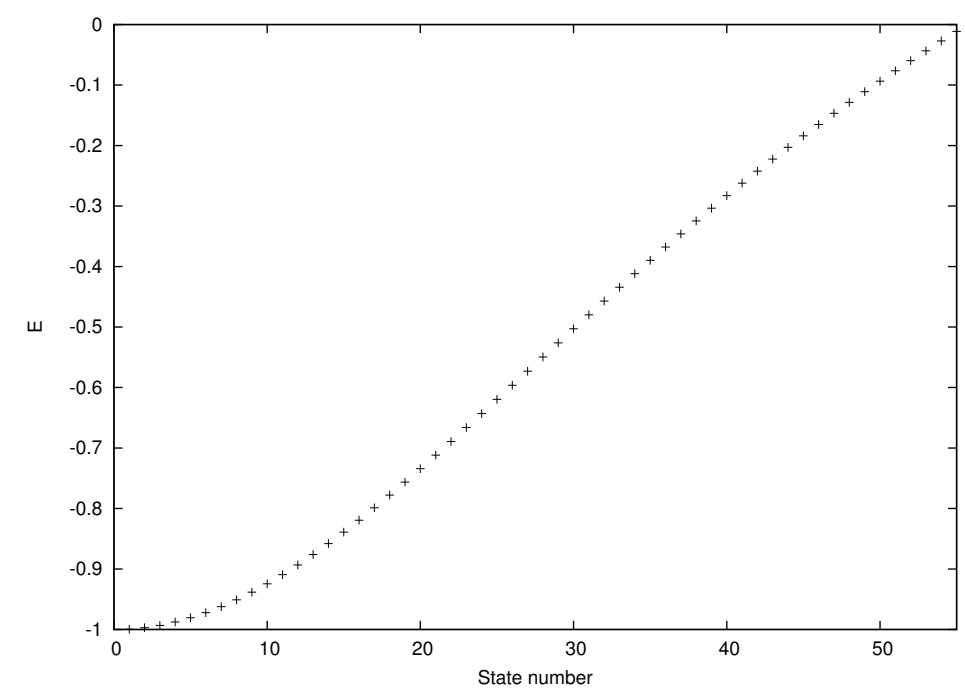

Fig. 1.3 Energies of the first 55 states of the pomeron with the fixed coupling. $t_{\max }=270$

\begin{tabular}{||c|c||c|c||}
\hline \multicolumn{1}{||c|}{$t_{\max }=90$} & \multicolumn{2}{c||}{$t_{\max }=270$} \\
\hline$-0.99335 \mathrm{E}+00$ & $0.25232 \mathrm{E}-03$ & $-0.99953 \mathrm{E}+00$ & $0.10250 \mathrm{E}-04$ \\
$-0.97347 \mathrm{E}+00$ & $0.98302 \mathrm{E}-03$ & $-0.99714 \mathrm{E}+00$ & $0.40871 \mathrm{E}-04$ \\
$-0.94143 \mathrm{E}+00$ & $0.21184 \mathrm{E}-02$ & $-0.99318 \mathrm{E}+00$ & $0.91482 \mathrm{E}-04$ \\
$-0.89868 \mathrm{E}+00$ & $0.35509 \mathrm{E}-02$ & $-0.98767 \mathrm{E}+00$ & $0.16146 \mathrm{E}-03$ \\
$-0.84704 \mathrm{E}+00$ & $0.51579 \mathrm{E}-02$ & $-0.98064 \mathrm{E}+00$ & $0.24995 \mathrm{E}-03$ \\
$-0.78851 \mathrm{E}+00$ & $0.68197 \mathrm{E}-02$ & $-0.97213 \mathrm{E}+00$ & $0.35588 \mathrm{E}-03$ \\
$-0.72505 \mathrm{E}+00$ & $0.84346 \mathrm{E}-02$ & $-0.96219 \mathrm{E}+00$ & $0.47801 \mathrm{E}-03$ \\
$-0.65850 \mathrm{E}+00$ & $0.99274 \mathrm{E}-02$ & $-0.95088 \mathrm{E}+00$ & $0.61492 \mathrm{E}-03$ \\
$-0.59048 \mathrm{E}+00$ & $0.11252 \mathrm{E}-01$ & $-0.93826 \mathrm{E}+00$ & $0.76509 \mathrm{E}-03$ \\
$-0.52233 \mathrm{E}+00$ & $0.12388 \mathrm{E}-01$ & $-0.92439 \mathrm{E}+00$ & $0.92686 \mathrm{E}-03$ \\
\hline
\end{tabular}

We also studied the behavior of the pomeron wave functions $\phi\left(q^{2}\right)$ as functions of $q^{2}$ for the three states with the lowest energy. It is shown in Fig. 1.4 Remarkably all three wave functions are very similar in their $q^{2}$ dependence in the low momentum region, but at larger momenta different states have a different number of nodes (zeros).

Let us analyze how the support in momentum space of the discrete states differ. This is best understood analyzing the rescaled eigenfunctions $\tilde{\phi}_{j}\left(q^{2}\right)$. The square of the first, second and sixth states with lowest energies are shown in Fig 1.5 . This behaviour is similar to the one found in 42$]$ where excited states show a clear shift towards higher values of $q^{2}$. One can see the dependence on the state number $\tilde{\phi}_{j}\left(q^{2}\right)$ of the position $q_{U V}(j)$ of the center of the most UV region of support. The 


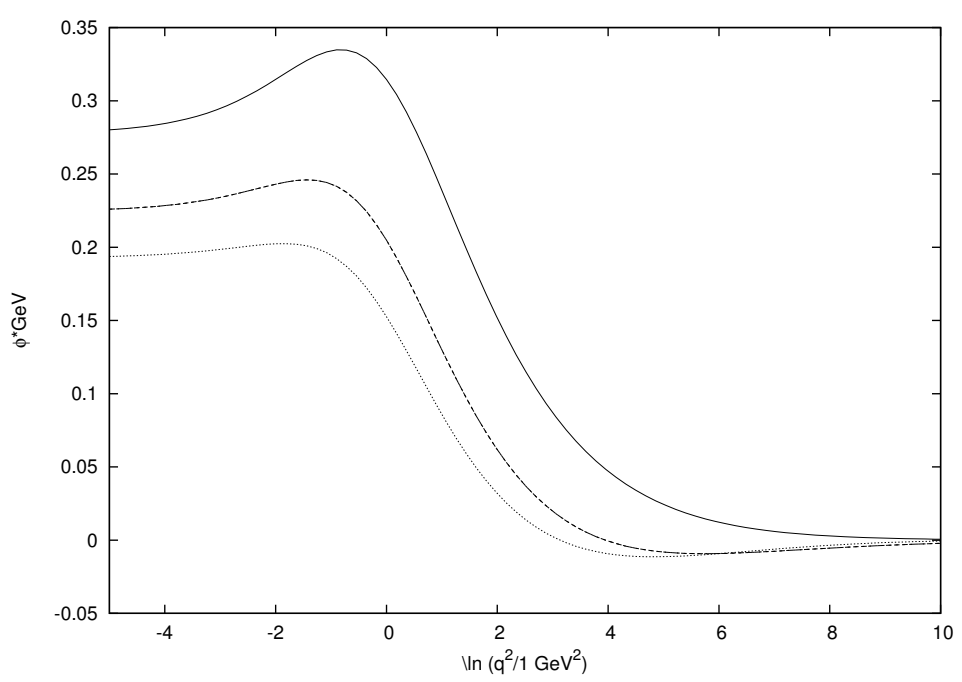

Fig. 1.4 Wave functions $\phi_{n}\left(q^{2}\right)$ as functions of $q^{2}$ for the first three states with the lowest energy. From top to bottom curves correspond to the number of state $n=1,2,3$

dependence is very well approximated with a simple linear fit. For example taking the first ten states one gets

$$
\ln q_{U V}^{2}(j) / \mathrm{GeV}^{2} \simeq-7.278+8.456 j .
$$

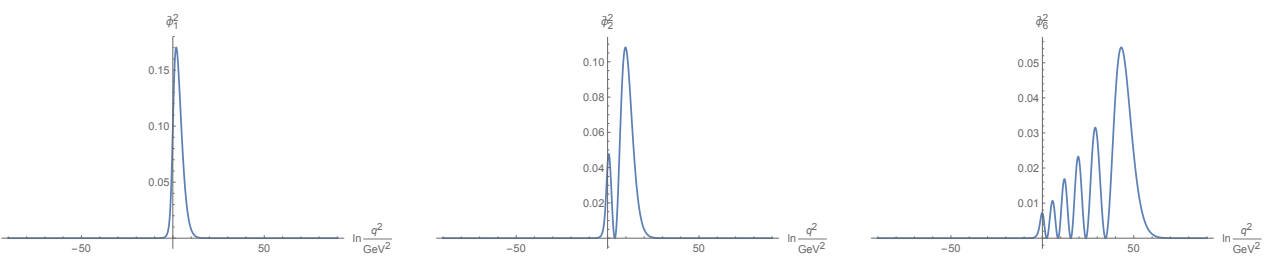

Fig. 1.5 Square of the pomeron eigenfunctions $\tilde{\phi}_{i}\left(q^{2}\right)$ for the first, second and sixth leading energy states. The region of highest momentum support is pushed rapidly towards the UV with the increasing order of the eigenstate.

Finally let us comment on the dependence on the mass parameter $\mathrm{m}$ which also appears inside the logarithmic dependence of the running coupling (keeping the same value of $\Lambda_{Q C D}$ ), and whose value fixes the order of the scale where $\alpha_{s}$ feezes. Lowering $m$ from $0.82 \mathrm{GeV}$ to $0.52 \mathrm{Gev}$, for the leading state the energy (=1-intercept $)$ increases in absolute value of about $20 \%$ while the slope changes much more, becoming almost 4 times larger, which is close to the well known value $0.25 \mathrm{GeV}^{-2}$. 


\subsubsection{Odderon}

Passing to the odderon let us first discuss the intercept. We have found that both with the running and fixed coupling constants energies lie on the cut starting at $E=0$. The density of states rises with $t_{\max }$. In particular with the running coupling the number of states with $E<0.02$ rises from 18 at $t_{\max }=90$ to 135 at $t_{\max }=270$. With the fixed coupling constant the density of states is considerably smaller and rises not so fast. At $t_{\max }=90$ we found only 5 states with $E<0.02$ and at $t_{\max }=270$ their number rises to 13 . Location of energies smaller than 0.02 for low-lying states for the running and fixed coupling constants is illustrated in Figs. 1.6 and 1.7 respectively.

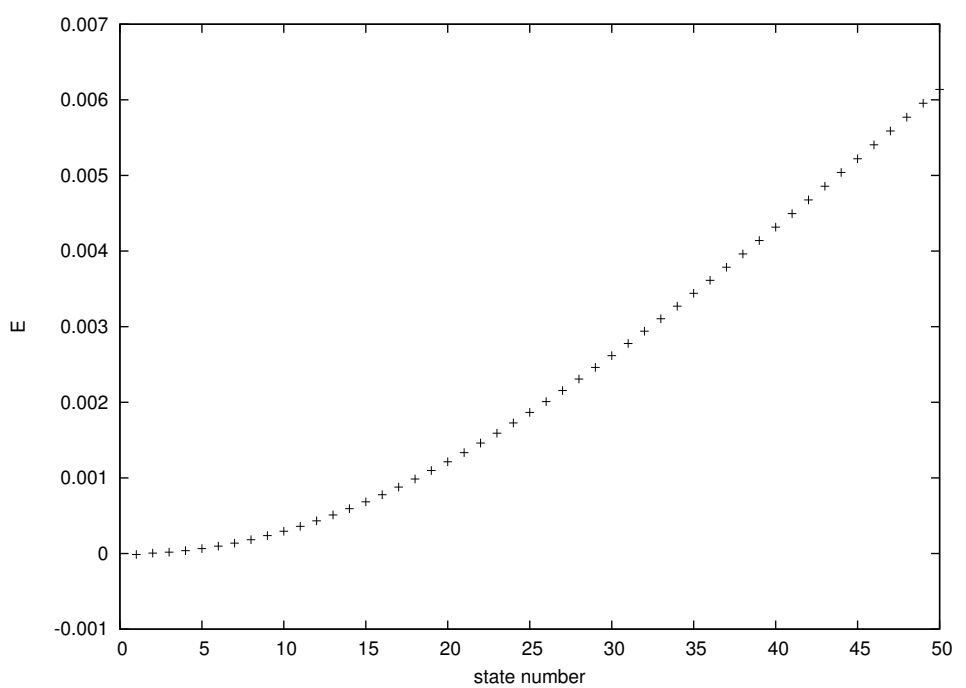

Fig. 1.6 Energies of the first 50 states of the odderon with the running coupling. $t_{\max }=270$

For the odderon we studied the character of the singularity of its spectrum at the branch-point $E=0$. It is revealed in the behavior of the amplitude governed by the odderon exchange at high $s$, Namely if the spectral density $\tau(E)$ has a singularity $E^{\beta}$ at $E=0$ the amplitude will diminish as $y^{-(1+\beta)}$ at large $y=\ln s$. In our calculations the continuous spectrum is approximated by a set of poles at $E_{i}, i=1,2,3, \ldots$ with very small distances from one another. We introduced the coupling $a_{i}$ of the $i$-th odderon state to the projectile or target taking for each of them a certain distribution of colour $\rho\left(q^{2}\right)=c \exp \left(-r_{0} q^{2}\right)$ with $r_{0}$ of the order of the proton radius. Then

$$
a_{i}=\sum_{j} \sqrt{w_{j} q_{j}} \rho\left(q_{j}^{2}\right) v_{j}^{(i)}
$$

where $v^{(i)}$ is the eigenvector for the $i$-th odderon (actually its wave function on the grid). The discrete spectral density is accordingly taken as $\tau_{i}=a_{i}^{2}$. With this we 


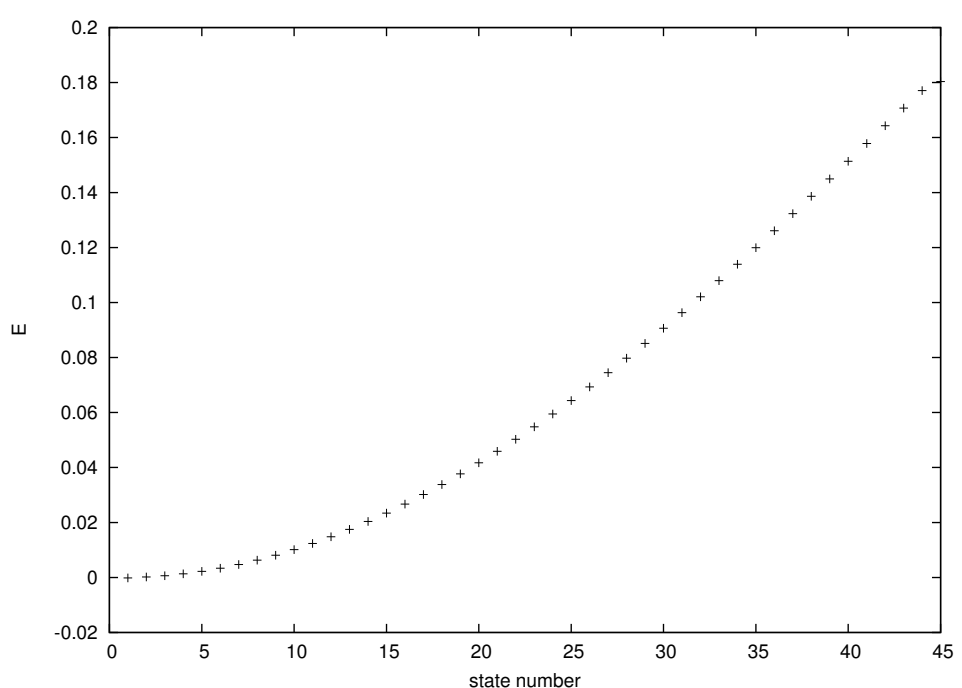

Fig. 1.7 Energies of the first 55 states of the odderon with the fixed coupling. $t_{\max }=270$

studied the $y$-dependence of the amplitude coming from the exchange of all odderon states

$$
\mathcal{A}(y)=\sum_{i} a_{i}^{2} e^{-E_{i} y} .
$$

The ratios $r(y)=\mathcal{A}(y) / \mathcal{A}(0)$ are plotted in Fig. 1.8 for the odderon with running and fixed coupling constants. It is clearly seen that running of the coupling makes the amplitude fall somewhat weaker at high $y$. This shows how important is important the incusion of running coupling effects for any quantitative estimate of an odderon exchange cross section. Fits to the curves give the behavior $\sim y^{-0.38}$ and $\sim y^{-0.86}$ for the running and fixed coupling respectively. This implies that the singularity at $E=0$ of the spectral density is stronger with the running coupling $E^{-0.62}$ than with the fixed one $E^{-0.14}$.

These results are qualitatively similar to the ones recently found using another prescription to introduce the running coupling [52].

\subsection{Conclusions}

We have focused on some aspects of QCD strong interactions involving the so called odderon exchange, which may be relevant in the scattering process in the Regge limit and also in presence of dense nuclear media, and are still posing challenging problems both theoretically and experimentally. It is still to be understood and investigated more at experimental level the difference at high energies in the scattering of $p p$ and $p \bar{p}$, even if new data started to arrive from the TOTEM experiment. Non perturbative physics and the uncertainties in non linear effects on parton distributions are main theoretical obstacles. 


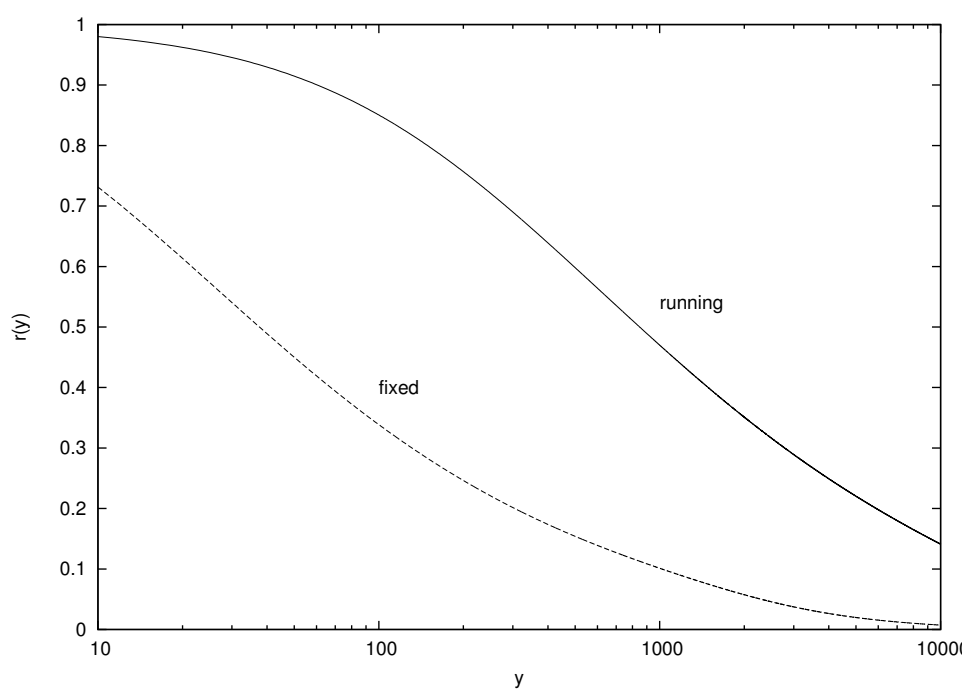

Fig. 1.8 The behavior of the amplitude with the odderon exchange $A(y)$ as a function of rapidity $y$. The upper and lower curves show the ratios $A(y) / A(0)$ with and without running of the coupling

We have discussed, in the context of perturbative QCD and BFKL physics, a path to improve the description of a family of known and leading odderon solutions (BLV) as well as more general BKP states in the LL approximation and large $N_{c}$ limit to account for running coupling effects. This is obtained requiring a match with standard one loop running coupling properties which leaves the freedom to model the IR non perturbative region and at the same time respects s-channel unitarity for the reggeization of the gluon at the base of the BFKL approach. This so called bootstrap approach was shown at perturbative level to reproduce running coupling properties of the NLL BFKL. For the pomeron channel we explicitly show that this procedures leads to a series of discrete leading poles with intercept greater than one, which accumulates at one.

For the odderon the inclusion of the running coupling makes more dense the states close to intercept one, where the cut continues to start. Therefore the rapidity dependence in the scattering amplitudes is affected making cross section larger, so that running coupling effects should not be neglected.

Pomeron and odderon fields can be elements of an effective description to study rapidity dependence and saturation effects in dense nuclear media or in extreme high energy scattering processes. Alternative descriptions to the reggeized gluon approach are the color dipole picture (large $N_{c}$ ) and the CGC approach. We have shown how to reproduces in the BFKL description the non linear evolution of the BFKL pomeron induced by the odderon splitting which was known from previous CGC studies. To derive in the BFKL framework also a non linear evolution for the odderon [21] the $V_{3 \rightarrow 5}$ reggeized gluon vertex should be computed. One of the main theoretical problems is that these effective theories do not take into account 
pomeron/odderon loops since only tree level effective equations are available. The loops were formally considered in the old days before the advent of QCD in the effective reggeon field theory where local fields for pomeron and odderon were considered. This description may be valid only for very large transverse distances. We have recalled how one can compute the critical properties of such a theory and extract the universal behavior (critical exponents) even if this regime if far from be probed by any experimental setup. It is interesting that there is a universal link with statistical mechanical systems, in particular with the out of equilibrium dynamics in generalized directed percolating systems.

Finally we note that in the LL generalized BFKL framework all the known gauge invariant effective vertices are build using the bootstrap condition, and involve always reggeized gluon trajectories and the real production part of the two gluon BFKL kernel. Therefore one can envisage a way to introduce also there the running coupling by the same procedure to take into account this part of the effects which would otherwise appear only at the level of the NLL approximation.

To conclude we want to stress the most important and seminal contributions made by Lev Lipatov in this field and discusses here. Apart from being one of the father-founders of the whole reggeized gluons approach, he specifically actively dealt with odderon and BKP states during his career with outstanding results. He studied the conformal invariance of the odderon wave function and formulated equations which later were used to find the JW-odderon. He discovered that in the planar (multicolor) limit the BKP equations become fully integrable and showed their relation to the non-compact Heisenberg spin chain, which formed the basis of the subsequent study of their properties. This was the first demonstration of integrability in gauge theories. Lev Lipatov was the first to discuss the influence of the running of the coupling and to conclude that it splits the negative cut in the energies of the pomeron into a sequence of poles, the results fully confirmed later irrespective of the way to introduce this running. Lev Lipatov was one of the authors to show the equivalence of the reggeized gluons and dipole approaches for the fan diagram BK evolution equation and in relation to this formulated the correct transition to the coordinate representation as the transition to "the Möbius representation". He was also one of the authors to investigate the next-to-leading order for the odderon structure, the result which will certainly play its role in future studies.

\subsection{Appendix. The slope}

The slope can be obtained starting from Eq. 1.80, expanding up to second order in $q$ the relation

$$
\left\langle\left(h_{q}-h_{0}\right)\right\rangle_{0}=\epsilon(q)-\epsilon(0),
$$

where the average is done on the eigenstates in the forward direction $(q=0)$ and according to the previous notation as in Eq. 1.82 ) we consider separate contributions 
and write

$$
h_{q}-h_{0}=W^{k i n}(q)+W^{q l}(q)+W^{s e p}(q) .
$$

First we consider the function $f(p)$ of Eq. 1.52 which is a kind of buiding block appearing in different instances. According to the decomposition of Eq. 1.99) in the first two orders of $q$ we get

$$
f\left(q_{1}\right)=f(l)\left[1+a_{1}\left((q l)+\frac{1}{4} q^{2}\right)+\frac{1}{2} a_{2}(q l)^{2}\right],
$$

where we denote with $q l=q \cdot l$ the scalar product of the two dimensional vectors, and

$$
a_{1}=\frac{1+\ln \left[\left(l^{2}+m^{2}\right) / \Lambda^{2}\right]}{f(l)}, \quad a_{2}=\frac{1}{\left(l^{2}+m^{2}\right) f(l)} .
$$

From this result one directly finds also $f\left(q_{2}\right), f\left(q_{1}^{\prime}\right)$ and $f\left(q_{2}^{\prime}\right)$ simply changing $l \rightarrow-l, l \rightarrow l^{\prime}$ and $l \rightarrow-l^{\prime}$ respectively. Coefficients $a_{1}(l)$ and $a_{2}(l)$ do not change under $l \rightarrow-l$. As in [51] we denote them simply as $a_{1}$ and $a_{2}$. Coefficients $a_{1}\left(l^{\prime}\right)$ and $a_{2}\left(l^{\prime}\right)$ will be denoted $a_{1}^{\prime}$ and $a_{2}^{\prime}$ Also

$$
f(q)=f(0)\left(1+a_{3} q^{2}\right), \quad a_{3}=\frac{1+\ln m^{2} / \Lambda^{2}}{f(0)} .
$$

Note also that $q_{1}-q_{1}^{\prime}=q_{2}^{\prime}-q_{2}=l-l^{\prime}$ do not depend on $q$.

\subsection{1 $W^{\text {kin }}$}

The kinetic term (1.81) contains two terms corresponding to trajectories $\omega_{1}$ and $\omega_{2}$. For the first part of $W^{k i n}$ coming from $\omega_{1}$ we find

$$
\begin{gathered}
W_{1}^{k i n}=\frac{1}{2} \int d^{2} l^{\prime} \frac{f(l)}{f\left(l^{\prime}\right) f\left(l-l^{\prime}\right)}\left[a_{1}\left((q l)+\frac{1}{4} q^{2}\right)+\frac{1}{2} a_{2}(q l)^{2}\right. \\
\left.-a_{1}^{\prime}\left(\left(q l^{\prime}\right)+\frac{1}{4} q^{2}\right)+\left(a_{1}^{\prime 2}-\frac{1}{2} a_{2}^{\prime}\right)\left(q l^{\prime}\right)^{2}-a_{1} a_{1}^{\prime}(q l)\left(q l^{\prime}\right)\right] .
\end{gathered}
$$

The second part of $W^{k i n}$ is found to be

$$
\begin{gathered}
W_{2}^{k i n}=\frac{1}{2} \int d^{2} l^{\prime} \frac{f(l)}{f\left(l^{\prime}\right) f\left(l-l^{\prime}\right)}\left[a_{1}\left(-(q l)+\frac{1}{4} q^{2}\right)+\frac{1}{2} a_{2}(q l)^{2}\right. \\
\left.-a_{1}^{\prime}\left(-\left(q l^{\prime}\right)+\frac{1}{4} q^{2}\right)+\left(a_{1}^{\prime 2}-\frac{1}{2} a_{2}^{\prime}\right)\left(q l^{\prime}\right)^{2}-a_{1} a_{1}^{\prime}(q l)\left(q l^{\prime}\right)\right] .
\end{gathered}
$$

In the total $W^{k i n}$ terms linear in $q$ cancel and quadratic ones are doubled. Thus at second order in $q$ we find

$$
W^{k i n}=\int d^{2} l^{\prime} \frac{f(l)}{f\left(l^{\prime}\right) f\left(l-l^{\prime}\right)}\left[\frac{1}{4} q^{2}\left(a_{1}-a_{1}^{\prime}\right)+\frac{1}{2} a_{2}(q l)^{2}+\left(a_{1}^{\prime 2}-\frac{1}{2} a_{2}^{\prime}\right)\left(q l^{\prime}\right)^{2}-a_{1} a_{1}^{\prime}(q l)\left(q l^{\prime}\right)\right] .
$$




\subsection{2 $W^{q l}$}

At finite $q$ this part is given by 1.83 . One finds at second order in $q$

$$
\sqrt{\frac{f\left(q_{1}\right)}{f\left(q_{2}\right)}}=1+a_{1}(q l)+\frac{1}{2} a_{1}^{2}(q l)^{2}, \quad \sqrt{\frac{f\left(q_{2}^{\prime}\right)}{f\left(q_{1}^{\prime}\right)}}=1-a_{1}^{\prime}\left(q l^{\prime}\right)+\frac{1}{2} a_{1}^{\prime 2}\left(q l^{\prime}\right)^{2} .
$$

and taking the product

$$
\sqrt{\frac{f\left(q_{1}\right) f\left(q_{2}^{\prime}\right)}{f\left(q_{2}\right) f\left(q_{1}^{\prime}\right)}}=1+a_{1}(q l)-a_{1}^{\prime}\left(q l^{\prime}\right)+\frac{1}{2}\left(a_{1}(q l)-a_{1}^{\prime}\left(q l^{\prime}\right)\right)^{2} .
$$

In the second term of $L$ in Eq. 1.83 we have to change $l \rightarrow-l$ and $l^{\prime} \rightarrow-l^{\prime}$. So the total $q$-dependent factor becomes

$$
2+\left(a_{1}(q l)-a_{1}^{\prime}\left(q l^{\prime}\right)\right)^{2}
$$

from which one finally reads at second order in $q$

$$
W^{q l}\left(l, l^{\prime}\right)=-\frac{\left(a_{1}(q l)-a_{1}^{\prime}\left(q l^{\prime}\right)\right)^{2}}{f\left(l-l^{\prime}\right)} .
$$

\subsection{3 $W^{\text {sep }}$}

The separable part of the interaction is given by 1.84 . Here one obtains in the straightforward manner

$$
\begin{gathered}
\frac{f(q)}{\sqrt{f(q 1) f\left(q_{2}\right) f\left(q^{\prime} 1\right) f\left(q_{2}^{\prime}\right)}} \\
=\frac{f(0)}{f(l) f\left(l^{\prime}\right)}\left[1+q^{2}\left(a_{3}-\frac{1}{4}\left(a_{1}+a_{1}^{\prime}\right)\right)-\frac{1}{2}\left(a_{2}-a_{1}^{2}\right)(q l)^{2}-\frac{1}{2}\left(a_{2}^{\prime}-a_{1}{ }^{2}\right)\left(q l^{\prime}\right)^{2}\right] .
\end{gathered}
$$

From this one gets at second order in $q$

$$
W^{s e p}\left(l, l^{\prime}\right)=\frac{f(0)}{f(l) f\left(l^{\prime}\right)}\left[q^{2}\left(a_{3}-\frac{1}{4}\left(a_{1}+a_{1}^{\prime}\right)\right)-\frac{1}{2}\left(a_{2}-a_{1}^{2}\right)(q l)^{2}-\frac{1}{2}\left(a_{2}^{\prime}-a_{1}^{\prime 2}\right)\left(q l^{\prime}\right)^{2}\right] .
$$

\subsubsection{Averaging in the state with $n=0$}

First task is to fix the normalization. Let

$$
\mathcal{N}=\int d^{2} q \phi^{2}\left(q^{2}\right)=\pi \int d q^{2} \phi^{2}\left(q^{2}\right)=\pi \int d t q^{2} \phi^{2}\left(q^{2}\right) .
$$

On the grid we get

$$
\mathcal{N}=\pi \sum_{i=0}^{N} w_{i} q_{i}^{2} \phi^{2}\left(q_{i}^{2}\right)=\pi \sum_{i=0}^{N} v_{i}^{2}
$$


where we used (1.105). The normalization can be fixed requiring

$$
\sum_{i=0}^{N} v_{i}^{2}=1
$$

in accordance with the standard programs for the search of eigenvalues and eigenvectors. This implies $\mathcal{N}=\pi$ and means that after the integration of our $W$ with the functions $\phi\left(q^{2}\right)$ one has to divide the result by $\pi$.

1. $\left\langle W^{k i n}\right\rangle$.

In this way we find

$$
\begin{gathered}
\left\langle W^{k i n}\right\rangle=\frac{1}{4} q^{2} \int d l^{2} \phi^{2}(l) \int d l^{\prime 2} \frac{f(l)}{f\left(l^{\prime}\right)} \\
\times\left[\left(\frac{1}{2}\left(a_{1}-a_{1}^{\prime}\right)+\frac{1}{2} a_{2} l^{2}+\left(a_{1}^{\prime 2}-\frac{1}{2} a_{2}^{\prime}\right) l^{\prime 2}\right) B_{0}\left(l^{2}, l^{\prime 2}\right)-a_{1} a_{1}^{\prime} l l^{\prime} B_{1}\left(l^{2}, l^{\prime 2}\right)\right] .
\end{gathered}
$$

Passing first to the integration over $t$ and $t^{\prime}$ and to summation on the grid we get

$$
\begin{gathered}
\left\langle W^{k i n}\right\rangle=\frac{1}{4} q^{2} \sum_{i, j} \phi^{2}\left(l_{i}\right) l_{i}^{2} l_{j}^{2} w_{i} w_{j} \frac{f\left(l_{i}\right)}{f\left(l_{j}\right)} \\
\times\left[\left(\frac{1}{2}\left(a_{1 i}-a_{1 j}\right)+\frac{1}{2} a_{2 i} l_{i}^{2}+\left(a_{1 j}^{2}-\frac{1}{2} a_{2 j}\right) l_{j}^{2}\right) B_{0}\left(l_{i}^{2}, l_{j}^{2}\right)-a_{1 i} a_{1 j} l_{i} l_{j} B_{1}\left(l_{i}^{2}, l_{j}^{2}\right)\right] .
\end{gathered}
$$

The final expression in terms of $v$ is obtained after using (1.105)

$$
\begin{gathered}
\left\langle W^{k i n}\right\rangle=\frac{1}{4} q^{2} \sum_{i, j} v_{i}^{2} l_{j}^{2} w_{j} \frac{f\left(l_{i}\right)}{f\left(l_{j}\right)} \\
\times\left[\left(\frac{1}{2}\left(a_{1 i}-a_{1 j}\right)+\frac{1}{2} a_{2 i} l_{i}^{2}+\left(a_{1 j}^{2}-\frac{1}{2} a_{2 j}\right) l_{j}^{2}\right) B_{0}\left(l_{i}^{2}, l_{j}^{2}\right)-a_{1 i} a_{1 j} l_{i} l_{j} B_{1}\left(l_{i}^{2}, l_{j}^{2}\right)\right] .
\end{gathered}
$$

2. $\left\langle W^{q l}\right\rangle$

Doing the angular integrations and dividing by $\pi$ we obtain

$$
\left\langle W^{q l}\right\rangle=-\frac{1}{4} q^{2} \int d l^{2} d l^{\prime 2} \phi(l) \phi\left(l^{\prime}\right)\left[\left(a_{1}^{2} l^{2}+a_{1}^{\prime 2} l^{\prime 2}\right) B_{0}\left(l^{2}, l^{\prime 2}\right)-2 a_{1} a_{1}^{\prime} l l^{\prime} B_{1}\left(l^{2}, l^{2}\right)\right] .
$$

In going to variables $t$ and $t^{\prime}$ and then to the summation on the grid, using (1.105)

$$
\left\langle W^{q l}\right\rangle=-\frac{1}{4} q^{2} \sum_{i, j} \sqrt{w_{i} w_{j}} l_{i} l_{j} v_{i} v_{j}\left[\left(a_{1 i}^{2} l_{i}^{2}+a_{1 j}^{2} l_{j}^{2}\right) B_{0}\left(l_{i}^{2}, l_{j}^{2}\right)-2 a_{1 i} a_{1 j} l_{i} l_{j} B_{1}\left(l_{i}^{2}, l_{j}^{2}\right)\right] .
$$

3. $\left\langle W^{s e p}\right\rangle$

Starting from the expression 1.129 we have

$$
\begin{gathered}
\left\langle W^{s e p}\right\rangle=\frac{1}{4 \pi} \int d l^{2} d l^{2} \phi(l) \phi\left(l^{\prime}\right) d \chi d \chi^{\prime} \frac{f(0)}{f(l) f\left(l^{\prime}\right)} \\
\times\left[q^{2}\left(a_{3}-\frac{1}{4}\left(a_{1}+a_{1}^{\prime}\right)\right)-\frac{1}{2}\left(a_{2}-a_{1}^{2}\right)(q l)^{2}-\frac{1}{2}\left(a_{2}^{\prime}-a_{1}^{\prime 2}\right)\left(q l^{\prime}\right)^{2}\right] .
\end{gathered}
$$


Here all the angular dependence is trivial. After angular integration terms no depending on the angles give $4 \pi^{2}$ and those containing $\cos ^{2} \chi$ or $\cos ^{2} \chi^{\prime}$ give $2 \pi^{2}$. So we find

$$
\left\langle W^{s e p}\right\rangle=\pi q^{2} \int d l^{2} d l^{\prime 2} \frac{\psi(l) \psi\left(l^{\prime}\right) f(0)}{f(l) f\left(l^{\prime}\right)}\left[a_{3}-\frac{1}{4}\left(a_{1}+a_{1}^{\prime}\right)-\frac{l^{2}}{4}\left(a_{2}-a_{1}^{2}\right)-\frac{l^{\prime 2}}{4}\left(a_{2}^{\prime}-a_{1}^{\prime 2}\right)\right] .
$$

Passing first to integration over $t$ and $t^{\prime}$ and then on the grid we finally find

$$
\left\langle W^{\text {sep }}\right\rangle=\pi q^{2} \sum_{i, j} \sqrt{w_{i} w_{j}} \frac{v_{i} v_{j} l_{i} l_{j} f(0)}{f\left(l_{i}\right) f\left(l_{j}\right)}\left[a_{3}-\frac{1}{4}\left(a_{1 i}+a_{1 j}\right)-\frac{l_{i}^{2}}{4}\left(a_{2 i}-a_{1 i}^{2}\right)-\frac{l_{j}^{2}}{4}\left(a_{2 j}-a_{1 j}^{2}\right)\right] .
$$

The final rescaled slope is given by

$$
\tilde{\alpha}^{\prime}=\frac{1}{q^{2}}\left\langle W^{k i n}+W^{q l}+W^{s e p}\right\rangle .
$$

\subsubsection{Fixed coupling}

For comparison it is instructive to consider the simple case of the fixed coupling with regularization in the infrared:

$$
f(q)=q^{2}+m^{2} .
$$

The energy $E$ is then related to $\epsilon$ as

$$
E=\frac{3 \alpha_{s}}{2 \pi^{2}} \epsilon
$$

where $\alpha_{s}$ is the fixed coupling constant. To eliminate dependence on it is convenient to measure its relation of the energy of the BFKL ground state $E_{B F K L}=$ $12 \alpha_{s} \ln 2 / \pi$. Then

$$
E=\epsilon \frac{\epsilon}{8 \pi \ln 2} E_{B F K L} .
$$

With 1.136 functions $B_{n}$ and $C_{0}$ become known analytically. In particular,

$$
\begin{gathered}
B_{0}\left(q_{1}^{2}, q_{2}^{2}\right)=\frac{2 \pi}{r}, \quad r^{2}=\left(q_{1}^{2}-q_{2}^{2}\right)^{2}+2 m^{2}\left(q_{1}^{2}+q_{2}^{2}\right)+m^{4}, \\
B_{1}\left(q_{1}^{2}, q_{2}^{2}\right)=\frac{2 \pi b}{r(a+r)}, \quad a=q_{1}^{2}+q_{2}^{2}+m^{2}, \quad b=2 q_{1} q_{2}, \\
B_{01}\left(q_{1}^{2}, q_{1}^{2}\right)=\frac{2 \pi}{b}\left(1-\frac{r}{a+b}\right)
\end{gathered}
$$

and

$$
C_{0}\left(q 2, q_{1}^{2}\right)=\frac{2 \pi}{r_{1}}, \quad r_{1}^{2}=\left(q^{2}+2 q_{1}^{2}+2 m^{2}\right)^{2}-4 q^{2} q_{1}^{2} .
$$

In the calculation of slopes we find

$$
a_{1}(q)=\frac{1}{f(q)}, \quad a_{2}=0, \quad a_{3}=\frac{1}{f(0)} .
$$




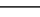




\section{Bibliography}

[1] C.Ewerz, (2003) arXiv: hep-ph/0306177.

[2] L.Lukaszuk and B.Nicolescu, Lett. Nuovo Cim. 8405 (1973).

[3] E.A.Kuraev, L.N.Lipatov, V.S.Fadin, Sov. Phys. JETP 45199 (1977).

[4] I.I.Balitski, L.N. Lipatov, Sov. J. Nucl. Phys. 28822 (1978).

[5] V.S.Fadin, L.N.Lipatov, Phys. Lett. B 429127 (1998).

[6] M.Ciafaloni, G.Camici, Phys. Lett. B 430349 (1998), [arXiv: hep-ph/9803389].

[7] J.Bartels, M.Wuesthoff, Z. Physik C 66157 (1995).

[8] A.Mueller, B.Patel, Nucl. Phys. B 425471 (1994).

[9] I.Balitski, Nucl. Phys. B 46399 (1996).

[10] Yu.V. Kovchegov, Phys. Rev. D 60034008 (1999).

[11] E.Ferreiro, E.Iancu, A.Leonidov, L. McLerran; Nucl. Phys.A 703489 (2002).

[12] M. Braun Eur. Phys. J. C 16 (2000) [hep-ph/0001268].

[13] J. Bartels, L. N. Lipatov and G. P. Vacca, Interactions of reggeized gluons in the Mobius representation, Nucl. Phys. B 706391 (2005) [hep-ph/0404110].

[14] J.Bartels, Nucl. Phys. B 175365 (1980).

[15] T. Jaroszewicz, Acta Phys. Polon. B 11965 (1980).

[16] J. Kwiecinski and M. Prasza lowicz, Phys. Lett. B 94413 (1980).

[17] L.N.Lipatov, JETP Lett. 59596 (1994).

[18] J.Wosiek and R.A.Janik, Phys. Rev. Lett. 792935 (1997).

[19] J. Bartels, L. N. Lipatov and G. P. Vacca, A New odderon solution in perturbative QCD, Phys. Lett. B 477178 (2000). [hep-ph/9912423].

[20] Y. V. Kovchegov, L. Szymanowski and S. Wallon, Phys. Lett. B 586267 (2004).

[21] Y. Hatta, E. Iancu, K. Itakura and L. McLerran, Odderon in the color glass condensate, Nucl. Phys. A 760172 (2005) [hep-ph/0501171].

[22] L. Motyka, Phys. Lett. B 637185 (2006), [hep-ph/0509270].

[23] J. Bartels, C. Ewerz. JHEP 9909026 (1999), [hep-ph/9908454].

[24] J. Bartels, M. A. Braun, D. Colferai and G. P. Vacca, Diffractive eta(c) photoproduction and electroproduction with the perturbative QCD odderon, Eur. Phys. J. C 20 323 (2001). [hep-ph/0102221].

[25] J. Bartels, V. S. Fadin, L. N. Lipatov and G. P. Vacca, NLO Corrections to the kernel of the BKP-equations, Nucl. Phys. B $\mathbf{8 6 7} 827$ (2013) [arXiv:1210.0797 [hep-ph]].

[26] J. Bartels and G. P. Vacca, Generalized Bootstrap Equations and possible implications for the NLO Odderon, Eur. Phys. J. C 732602 (2013) [arXiv:1307.3985 [hep-th]]. 
[27] J. Bartels, M. A. Braun and G. P. Vacca, The Process $\gamma^{*}+p-i \eta_{c}+X: A$ Test for the perturbative QCD odderon, Eur. Phys. J. C 33511 (2004) [hep-ph/0304160].

[28] G.Antchev et al. (TOTEM collab), [arxiv:1812.08610 [hep-ex]]

[29] V.M.Abazov et al. (D0 collab.), Phys. Rev. G 86012009 (2012), [hep-ex/1206.0687]

[30] V. A. Khoze, A. D. Martin, and M. G. Ryskin, Phys. Rev. D 97 no. 3, 034019, [arXiv:1712.00325 [hep-ph]].

[31] V. A. Khoze, A. D. Martin, and M. G. Ryskin, Eur. Phys. J. C 74 no. 2, (2014) 2756 (2018), [arXiv:1312.3851 [hep-ph]].

[32] E. Martynov and B. Nicolescu, Phys. Lett. B 778 (2018) 414-418, arXiv:1711.03288 [hep-ph].

[33] V. A. Abramovsky, V. N. Gribov and O. V. Kancheli, Character of Inclusive Spectra and Fluctuations Produced in Inelastic Processes by Multi - Pomeron Exchange, Yad. Fiz. 18595 (1973), [ Sov. J. Nucl. Phys. 18308 (1974) ].

[34] J. Bartels, M. Salvadore and G. P. Vacca, AGK cutting rules and multiple scattering in hadronic collisions, Eur. Phys. J. C 4253 (2005). [hep-ph/0503049].

[35] J. Bartels, L. N. Lipatov and G. P. Vacca, Interactions of reggeized gluons in the Mobius representation, Nucl. Phys. B 706391 (2005). [hep-ph/0404110].

[36] J. Bartels, L. N. Lipatov, M. Salvadore and G. P. Vacca, Deformed spectral representation of the BFKL kernel and the bootstrap for gluon reggeization, Nucl. Phys. B 72653 (2005). [hep-ph/0506235].

[37] M. A. Braun and G. P. Vacca, Triple pomeron vertex in the limit $N(c)-i$ infinity, Eur. Phys. J. C 6147 (1999). [hep-ph/9711486].

[38] J. Bartels, C. Contreras and G. P. Vacca, Could reggeon field theory be an effective theory for QCD in the Regge limit?, JHEP 1603201 (2016) [arXiv:1512.07182 [hepth]].

[39] J. Bartels, C. Contreras and G. P. Vacca, Pomeron - Odderon interactions in a reggeon field theory, Phys. Rev. D 95 no.1, 014013 (2017) [arXiv:1608.08836 [hep-th]].

[40] L.N.Lipatov, Sov. Phys. JETP 63904 (1986).

[41] H,Kowalski. L.N.Lipatov, D.S.Ross, Eur. Phys. J. C 74 no. 6, 2919 (2014)

[42] H,Kowalski. L.N.Lipatov, D.S.Ross, Eur. Phys. J. C 76 1-23 (2016)

[43] H,Kowalski. L.N.Lipatov, D.S.Ross, G.Watt, Eur. Phys. J. C 70983 (2010)

[44] J. Bartels, C. Contreras and G. P. Vacca, A functional RG approach for the BFKL Pomeron, JHEP 1901004 (2019) [arXiv:1808.07517 [hep-ph]].

[45] M.A.Braun, Phys. Lett., B 345 (1995) 155.

[46] M. Braun and G. P. Vacca, The 2nd order corrections to the interaction of two Reggeized gluons from the bootstrap, Phys. Lett. B 454319 (1999) [hep-ph/9810454].

[47] M. Braun and G. P. Vacca, The Bootstrap for impact factors and the gluon wave function, Phys. Lett. B 477156 (2000) [hep-ph/9910432].

[48] V. S. Fadin and A. Papa, A Proof of fulfillment of the strong bootstrap condition, Nucl. Phys. B 640309 (2002) [hep-ph/0206079].

[49] M.A.Braun, Eur. Phys. J. C 5359 (2008).

[50] G. P. Vacca, Properties of a family of $n$ reggeized gluon states in multicolor QCD, Phys. Lett. B 489337 (2000) [hep-ph/0007067].

[51] M. Braun, G. P. Vacca and G. Venturi, Properties of the hard pomeron with a running coupling constant and the high-energy scattering, Phys. Lett. B 388823 (1996) [hepph/9605304]. 
[52] J. Bartels, C. Contreras and G. P. Vacca, The Odderon in QCD with running coupling, arXiv:1910.04588 [hep-th]. 